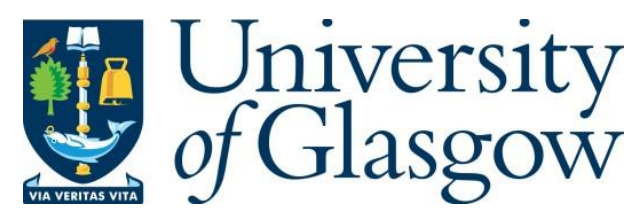

Reddy, N. H. and Saxena, P. (2017) Limit points in the free inflation of a magnetoelastic toroidal membrane. International Journal of Non-Linear Mechanics, 95, pp. 248-263.

There may be differences between this version and the published version. You are advised to consult the publisher's version if you wish to cite from it.

http://eprints.gla.ac.uk/174825/

Deposited on: 12 December 2018

Enlighten - Research publications by members of the University of Glasgow

http://eprints.gla.ac.uk 


\title{
Limit points in the free inflation of a magnetoelastic toroidal membrane
}

\author{
Narravula Harshavardhan Reddy, Prashant Saxena \\ Department of Mechanical \& Aerospace Engineering, Indian Institute of Technology Hyderabad \\ Kandi, Sangareddy 502285, Telangana, India
}

\begin{abstract}
One common phenomenon native to inflation of membranes is the elastic limit-point instability-a bifurcation point at which the membrane begins to deform enormously at the slightest increase of pressure. In the case of magnetoelastic materials, there is another possible phenomenon which we call magnetic limitpoint instability, a state referring to the non-existence of an equilibrium state - either stable or unstable. In this work, we are concerned with such instabilities in an incompressible isotropic magnetoelastic toroidal membrane with an initial circular cross-section. A non-uniform magnetic field is generated using a circular current carrying loop placed inside the membrane in addition to inflation by a uniform hydrostatic pressure. An energy formulation based on magnetization is used to model the magneto-mechanical coupling along with a Mooney-Rivlin constitutive model for the elastic strain energy density. Computations show that the magnetic field strongly influences the location of elastic limit points and in some cases can cause them to vanish. Multiple equilibrium states are obtained as solutions of the governing equations and a criterion based on second variation is employed to determine their stability. Existence and dependence of magnetic limit point on the magnetic field is demonstrated. While the quantitative results obtained here are specific to the toroidal geometry, the deformation behaviour can be generalised to any magnetoelastic membrane.
\end{abstract}

Keywords: magnetoelasticity; finite deformation; membrane; limit point

Mathematics Subject Classification: 74B20, 74F15, 74G65, 74K15

Note: This is an author generated version of the article published in the "International Journal of Non-Linear Mechanics" (2017). http://dx.doi.org/10.1016/j.ijnonlinmec.2017.06.017

\section{Contents}

1 Introduction 2

2 General deformation $\quad 4$

2.1 Kinematics of deformation . . . . . . . . . . . . . . . . . . . . . 4

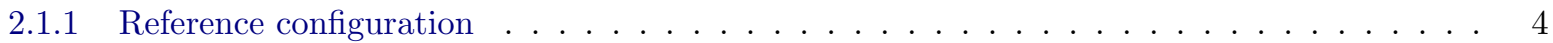

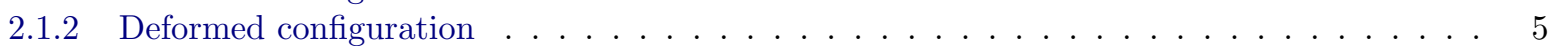

2.2 Energy considerations . . . . . . . . . . . . . . . . . . . . . 6

2.2.1 Total potential energy . . . . . . . . . . . . . . . . . . . . . 6

2.2 .2 Variation in elastic strain energy . . . . . . . . . . . . . . . . . . . 7

2.2.3 Variation in pressure energy . . . . . . . . . . . . . . . . . . . . . 8

2.2 .4 Variation in energy of the magnetic field . . . . . . . . . . . . . . . . . . . . . 8

2.3 Governing equations . . . . . . . . . . . . . . . . . . . . . . . . 10

2.4 Solution Procedure . . . . . . . . . . . . . . . . . . . . . . . . . . . 11

2.5 Second Variation . . . . . . . . . . . . . . . . . . . . . . . . . 12

3 Parametric deformation $\quad 12$

4 Cauchy stresses $\quad 15$

5 Results and discussion $\quad 16$

5.1 Validating the formulation . . . . . . . . . . . . . . . . . . . . . 16

5.2 Comparison of general and parametric deformations . . . . . . . . . . . . . . . . . 16

5.3 Limit points . . . . . . . . . . . . . . . . . . . . . . . . . 18

5.4 Multiple equilibrium states . . . . . . . . . . . . . . . . . . . . . . . 23

5.5 Stationary current carrying loop . . . . . . . . . . . . . . . . . . . . . 23 


\section{Introduction}

Nonlinear elastic membranes find numerous applications in the fields of structural and aerospace engineering, and safety. Inflatable balloons, roof tops, air bags, diaphragm valves, and protecting/cushioning membranes are some examples. Many biological materials including cell membranes, tissues, arterial walls also fall in this material category (see (Humphrey, 2003)). Study of the mechanical behaviour of membranes should account for the nonlinearity in their geometry as well as the material models. Several extensive studies have been carried out, for example on the inflation of elastic membranes by Wineman (1976), Naghdi and Tang (1977), Fulton and Simmonds (1986), Khayat and Derdouri (1995), Jiang and Haddow (1995), Bonet et al. (2000), Patil et al. (2014), and on contact problems by Feng and Yang (1973), and Charrier et al. (1989).

Axisymmetric deformations of membranes with simply connected geometries were studied analytically through a direct integration method (Yang and Feng, 1970) while those of non-simply connected geometries, such as a toroid, had to be studied through perturbation methods and certain approximations. Studying non-axisymmetric problems can be even more challenging but they are prevalent in practical applications, for example, see the papers by Grossman (1991a,b, 1994) on the rim supports for inflatable reflectors for space applications. Khayat and Derdouri $(1994 \mathrm{a}, \mathrm{b})$ studied inflation of cylindrical membranes for both axisymmetric and non-axisymmetric cases. An elaborate literature survey on this topic can be found in the paper by Jenkins and Leonard (1991).

Axisymmetric deformation of toroidal membranes with initial circular cross-section under uniform internal pressure has been interesting to researchers for more than half a century due to the complexity in their geometry and mechanical behaviour. To quote a few earlier studies, Sanders and Liepins (1963) employed direct integration technique to solve the governing equations for a linearly elastic torus and Jordan (1962) studied the nonlinear membrane deformation with linear constitutive relations using numerical integration. Early instances of continuum mechanics approach can be seen in the work of Kydoniefs and Spencer (1965) who used perturbation techniques, Hill (1980) who used numerical approach, Bonadies (1987) for asymptotic solutions, and Xin-chun and Chang-jun (1991) who used shooting method followed by Newton-Raphson method for solving the two-point boundary value problem in studies of the inflation of a completely non-linear elastic incompressible torus. However, their studies were limited to the assumption that the ratio of radius of the generating circle to the overall radius remains very small throughout the inflation process. The same assumption has been made by Papargyri-Pegiou (1995) and Papargyri-Pegiou and Stavrakakis (2000) for obtaining analytical and numerical solutions. Some examples of studies without this assumption include Fried (1982) who used finite element approach for large deformation of rubber membranes and Li and Steigmann (1995) who used tension field theory and Newton-Raphson method to numerically integrate the governing equations. An instance of finite element approach for large deformations can be found in a recent study by Papargyri-Beskou (2005). Tamadapu and DasGupta $(2012$, 2013, 2014a,b) have worked on inflation of incompressible toroidal membranes in which they also proposed directly integrating the system of second order ordinary differential governing equations. The boundary value problem is converted to an initial value problem by employing shooting method and a solution for the resulting problem is searched using Nelder-Meads optimization technique. We use the same solution procedure in our current study. We also check the validity of the old assumption that the cross-section remains circular throughout the inflation process and simplify the mathematics involved for the cases where it holds.

Magnetoelastic polymers have the ability to respond mechanically to an applied magnetic field and viceversa. Fabrication and experimental characterization of such magnetoelastic elastomers are discussed by, to quote a few, Bossis et al. (2001), Farshad and Benine (2004), Gong et al. (2005), Wang et al. (2006), and Krautz et al. (2017). They typically contain numerous tiny ferromagnetic particles (like iron) embedded in a polymer matrix such as rubber. In the presence of an external magnetic field, individual magnetization vectors of the ferromagnetic particles align with the applied field and the resulting interaction leads to a change in the observed macroscopic stiffness and dimensions of the polymer (Böse and Röder, 2009; Ginder et al., 2002). This possibility to alter mechanical properties has found applications in sensors and actuators, active vibration control and waveguides (for example, see the works of, Ginder et al. (1999, 2001), Holmes et al. (2012), Li and Zhang (2008), Böse et al. (2012), Keh et al. (2013), and Saxena (2017)).

Theoretical framework for the study of electromagnetic interactions in deformable continua has been available for over five decades. The classical works of Truesdell and Toupin (1960), Tiersten (1964), Maugin and Eringen (1972), Pao and Yeh (1973), and Pao (1978) utilise the conservation laws for continua to arrive at the required governing equations of the problem. Tiersten (1965) and Brown (1966) take a Lagrangian approach to 
the problem by the minimisation of a total potential energy function. These classical theories have been used in the recent years to account for large deformations and nonlinear magneto-mechanical interaction in order to study magnetoelastic polymers. Brigadnov and Dorfmann (2003), and Dorfmann and Ogden (2003) proposed a formulation based on a total free energy density that is a function of deformation and the magnetic flux density. It was later shown by Dorfmann and Ogden (2005) that magnetic field or magnetization vector can also be used as an independent variable in the same formulation. Steigmann (2004) used the magnetic field intensity as an independent variable and was among the earlier works on magnetoelastic membranes. Kankanala and Triantafyllidis (2004) extended the work of Brown (1966) and used the variational formulation to arrive at similar governing equations. They claim that magnetization per unit mass can be a better choice for an independent variable along with deformation gradient because, unlike magnetic flux or intensity, it vanishes outside the material. Their work (with magnetization as an independent variable in free energy density function) has later been utilised by Barham et al. (2007, 2008) for studying thin magnetoelastic membranes. We follow the same formulation in the current study.

Several studies in magneto-mechanics have explicitly focussed on the modelling of the magnetoelastic polymers. By appropriately considering the micromechanical constitution of magnetoelastic polymers, formulations for coupled field equations using homogenisation techniques have been proposed by Castañeda and Galipeau (2011); Galipeau and Castañeda (2013) and Chatzigeorgiou et al. (2014), among others. Phenomenon of energydissipation due to viscoelasticity has been modelled by Saxena et al. (2013), Ethiraj and Miehe (2016), and Haldar et al. (2016); and anisotropic structure of magnetoelastic polymers has been accounted in the models by Bustamante (2010), Danas et al. (2012a), and Saxena et al. (2014). However, in this work, we restrict our attention to isotropic and conservative magnetoelastic systems. It should be noted that a parallel track of research exists in the field of nonlinear electroelasticity that is largely motivated by electroactive polymers. Although the constitution of those materials is quite different, the mathematical formulation is very similar, for example, see the papers by Dorfmann and Ogden (2005), McMeeking and Landis (2005), and Castañeda and Siboni (2012). For an exhaustive survey on electro- and magneto-mechanics, we refer to the recent books on the subject by Ogden and Steigmann (2011), and Dorfmann and Ogden (2014).

In the case of membranes, limit point elastic instability is an important phenomenon to look for during the inflation process. This is the critical point (also referred to as bifurcation point by some authors) after which the membrane begins to deform enormously even for a slightest increase in pressure. Several examples of the study of these critical points are available in the literature. For example, Benedict et al. (1979) studied limit point pressures in nonlinear elastic tubes, Khayat et al., 1992 studied deformation of cylindrical membranes with special emphasis on bulging after the critical point. They also pointed out the importance of constitutive models, inflation conditions and geometries in understanding the phenomenon. Needleman (1977) and then later Patil et al. (2015) studied bifurcations in equilibrium paths for spherical and cylindrical membranes, respectively. Increasing surface area and decreasing thickness call for a local maximum in the inflating pressure. Beyond this point, multiple equilibrium states are possible for the membrane (generally, only the stable ones are shown in a pressure-stretch plot). Hence, in practical applications, it is crucial to know the limit point or the critical pressure apriori for the membrane and the given constraints. At some point beyond the critical state, the pressure starts to increase once again due to strain hardening of the material. Alexander (1971) has demonstrated this behaviour in spherical rubber balloons using the form of total energy function at equilibrium states. It should also be noted that the character of this instability is decided by the constitutive model used (Kanner and Horgan, 2007; Khayat et al., 1992). Inflation curve of a neo-Hookean material has a peak at the critical point followed by a continuous drop in the pressure. Strain hardening is not predicted by this model whereas, depending on the material parameters, Mooney-Rivlin constitutive model does predict this phenomenon (characterized by a local minimum) observed in experiments. Recently, Tamadapu et al. (2013) have studied the material and geometric aspects of the elastic instability for a few axisymmetric problems, including a torus. They found that the value of limit point pressure can be estimated from the geometric and material parameters alone. Such geometrical connections to the limit point behaviour is beyond the scope of our current study. We do, however, encounter additional limit points due to magnetic field and address them in this work.

Recently, Raikher et al. (2008) have performed experiments on the deformation of a circular magnetoelastic membrane under a uniform magnetic field. In the parallel field of electroelasticity, Fox and Goulbourne (2008) study the displacement of a circular membrane and its dependence on applied potential difference across the membrane and its frequency, Fox and Goulbourne (2009) and Tews et al. (2003) present pressure vs chamber volume characteristics, Keplinger et al. (2012) and Li et al. (2013) study the snap-through instability in the inflation process. However, none of these studies considered wrinkling or limit point instabilities in membranes. Barham et al. (2008) evaluated the solution for inflation of a circular magnetoelastic membrane under the influence of a stationary magnetic dipole. The arrangement is such that upon inflation, the membrane gets 
closer to the dipole. They observed that, for a given dipole strength, when the dipole is sufficiently close to the membrane, stable and unstable equilibria merge and cease to exist thereafter. We look for similar phenomenon in the present study and we term such a state 'magnetic limit point instability'.

Remainder of this article is organised as follows: Section 2 discusses the kinematics of deformation, derivation of governing equations and the solution procedure for the inflation of a toroidal membrane without any constraints on the deformed shape, which we call 'general deformation'. Section 3 extends the same to a special case where the cross-section of the membrane is assumed to remain circular throughout the inflation process (with the radius allowed to change). We name it 'parametric deformation' since two parameters are sufficient to describe any deformed state. Section 4 discusses the Cauchy stresses that arise in the problem and in Section 5 , we present and discuss our numerical results. The conclusions of this study are reported in Section 6 .

\section{General deformation}

Figure 1 shows the incompressible nonlinear magnetoelastic toroidal membrane with initial circular cross-section in its undeformed and deformed states. We restrict the solution space to the deformations that are symmetric with respect to $Y^{1}-Y^{2}$ plane and rotations about the $Y^{3}$ axis. We also note that the two sets of Euclidean coordinates in Figure 1: $\left(Y^{1}, Y^{2}, Y^{3}\right)$ and $\left(y^{1}, y^{2}, y^{3}\right)$ are in the same directions.

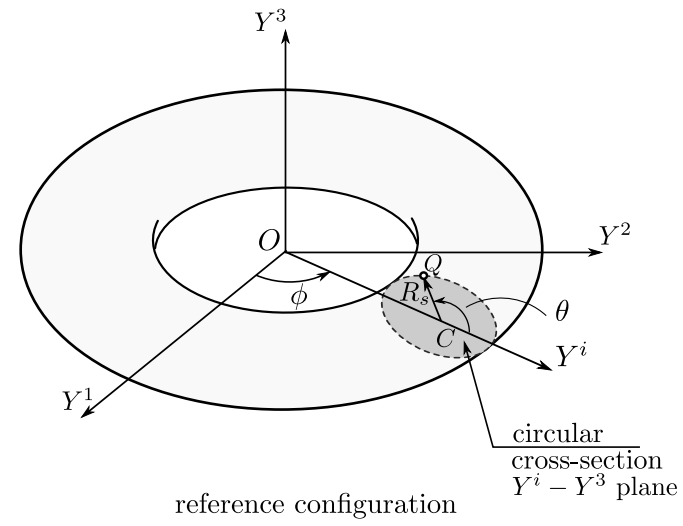

(a)

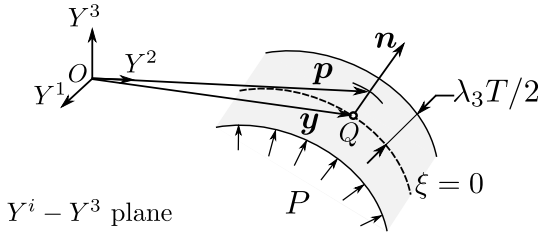

flesh of the deformed membrane

(b)

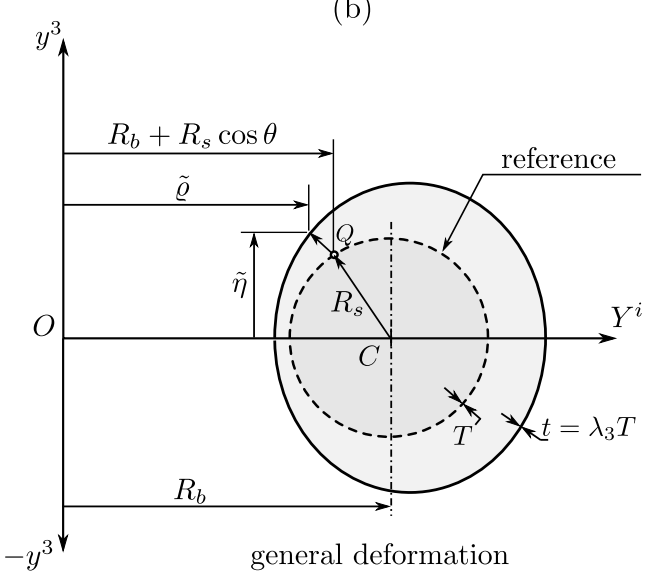

(c)

Figure 1: Toroidal membrane (a) before deformation with a circular cross-section highlighted, (b) a slice of the membrane thickness acted upon by an internal gas pressure $P$, and (c) the cross-section after general deformation illustrated through a point $Q$ on $Y^{i}-Y^{3}$ plane. The membrane at any instant is symmetric about $Y^{1}-Y^{2}$ plane and about the $Y^{3}$ axis. $T$ is the undeformed membrane thickness.

\section{$2.1 \quad$ Kinematics of deformation}

\subsubsection{Reference configuration}

The position vector of a point in the flesh of the undeformed toroidal membrane $\boldsymbol{X}$ is given as

$$
\boldsymbol{X}(\theta, \phi)=\left(R_{b}+\left(R_{s}+\xi\right) \cos \theta\right) \cos \phi \boldsymbol{E}_{1}+\left(R_{b}+\left(R_{s}+\xi\right) \cos \theta\right) \sin \phi \boldsymbol{E}_{2}+\left(R_{s}+\xi\right) \sin \theta \boldsymbol{E}_{3},
$$

where $R_{b}$ is the radius of revolution of the centre of the initial circular cross-section with radius $R_{s}$ and $\xi$ is the distance of the point from the mid-surface (given by $\xi=0$ ) of the membrane along the radius (see Figure 1; 
(Spain, 1965, Ch. 6)), and $\left\{\boldsymbol{E}_{i}\right\}$ are the orthonormal bases vectors corresponding to the $Y^{i}$ coordinate system. Now, the bases in the curvilinear system $(\theta, \phi, \xi)$ at a point $\boldsymbol{X}$ become

$$
\boldsymbol{G}_{i}=\frac{\partial \boldsymbol{X}}{\partial X^{i}}, \quad \text { where }\left(X^{1}, X^{2}, X^{3}\right)=(\theta, \phi, \xi)
$$

The components of the covariant metric tensor $G_{i j}=\boldsymbol{G}_{i} \cdot \boldsymbol{G}_{j}$ for the thin membrane $\left(\xi \ll R_{s}<R_{b}\right)$ are as follows

$$
G_{i j}=\left(\begin{array}{ccc}
R_{s}^{2} & 0 & 0 \\
0 & \left(R_{b}+R_{s} \cos \theta\right)^{2} & 0 \\
0 & 0 & 1
\end{array}\right) .
$$

Elements of a metric tensor denote the distortion of the coordinates when transformed from the Cartesian to the curvilinear system e.g., here, square of a differential length in the Cartesian system becomes $G_{11}(\mathrm{~d} \theta)^{2}+$ $G_{22}(\mathrm{~d} \phi)^{2}+G_{33}(\mathrm{~d} \xi)^{2}$ in the new system. Similarly, a differential area on the mid-surface in the new system is $G \mathrm{~d} \theta \mathrm{d} \phi$ where $G=\operatorname{det}\left(G_{i j}\right), i, j=1$ to 2 .

\subsubsection{Deformed configuration}

Let the position vector of a point anywhere in the flesh of the membrane be denoted by $\boldsymbol{p}$ and that of its projection, along the normal vector $\boldsymbol{n}$, onto the mid-surface by $\boldsymbol{y}$. The covariant metric tensor is denoted by $\tilde{g}_{i j}$ while

$$
\lambda_{3}(\theta)=t / T
$$

denotes the change in thickness during the deformation where $t$ and $T$ are the values of membrane thickness in the current and reference configurations, respectively.

Figure $1 \mathrm{~b}$ shows a slice of the membrane thickness around a point $Q$ in its deformed state. A point within the membrane which was originally at a distance $\xi$ from the mid-surface is now at a distance $\lambda_{3} \xi$ in the deformed state. By extension, the inner $(\xi=-T / 2)$ and outer $(\xi=+T / 2)$ surfaces are now at a distance $\lambda_{3} T / 2$ from the mid-surface $(\xi=0)$.

Following similar procedure as in Section 2.1.1,

$$
\begin{gathered}
p^{i}=y^{i}+\xi \lambda_{3} n^{i}, \\
\text { with } \quad y^{1}=\tilde{\varrho}(\theta) \cos \phi, \quad y^{2}=\tilde{\varrho}(\theta) \sin \phi, \quad y^{3}=\tilde{\eta}(\theta) .
\end{gathered}
$$

where $\varrho$ represents the horizontal distance, from origin $O$, of the point which was originally at the meridional coordinate $\theta$ in its undeformed state, and $\tilde{\eta}$ represents the vertical distance (see Figure 1 ). Tangent vectors at the points $\boldsymbol{y}$ and $\boldsymbol{p}$ respectively are given as

$$
\boldsymbol{g}_{i}=\frac{\partial \boldsymbol{y}}{\partial X^{i}} \text { and } \tilde{\boldsymbol{g}}_{i}=\frac{\partial \boldsymbol{p}}{\partial X^{i}}
$$

Since the normal vector is perpendicular to the tangent vectors $\boldsymbol{g}_{1}$ and $\boldsymbol{g}_{2}$,

$$
\begin{gathered}
\boldsymbol{n}=\frac{-\boldsymbol{g}_{1} \times \boldsymbol{g}_{2}}{\left|\boldsymbol{g}_{1} \times \boldsymbol{g}_{2}\right|}=-\frac{1}{\tilde{\varrho} \sqrt{\tilde{\varrho}^{\prime 2}+\tilde{\eta}^{\prime 2}}}\left(\boldsymbol{y}_{, 1} \times \boldsymbol{y}_{, 2}\right), \\
\text { or } \quad n^{i}=-\frac{1}{\sqrt{g}} \epsilon_{i j k} y_{, 1}^{j} y_{, 2}^{k}
\end{gathered}
$$

where $(\cdot)^{\prime}=\mathrm{d}(\cdot) / \mathrm{d} \theta$ everywhere, $\epsilon_{i j k}$ is a permutation symbol and $\sqrt{g}=\tilde{\varrho} \sqrt{\tilde{\varrho}^{\prime 2}+\tilde{\eta}^{\prime 2}}$. The negative sign here is due to the sense of $\theta$ and $\phi$ chosen in the problem. Also, expanding the above expression, we get

$$
\begin{aligned}
& n^{1}=\frac{1}{\sqrt{g}}\left(y_{, 1}^{3} y_{, 2}^{2}-y_{, 1}^{2} y_{, 2}^{3}\right)=\frac{\tilde{\varrho} \tilde{\eta}^{\prime} \cos \phi}{\sqrt{g}} \\
& n^{2}=\frac{1}{\sqrt{g}}\left(y_{, 1}^{1} y_{, 2}^{3}-y_{, 1}^{3} y_{, 2}^{1}\right)=\frac{\tilde{\varrho} \tilde{\eta}^{\prime} \sin \phi}{\sqrt{g}} \\
& n^{3}=\frac{1}{\sqrt{g}}\left(y_{, 1}^{1} y_{, 2}^{2}-y_{, 1}^{2} y_{, 2}^{1}\right)=\frac{-\tilde{\varrho} \tilde{\varrho}^{\prime}}{\sqrt{g}} .
\end{aligned}
$$

Components of the covariant metric tensor in the deformed state are as follows.

$$
\tilde{g}_{i j}=\tilde{\boldsymbol{g}}_{i} \cdot \tilde{\boldsymbol{g}}_{j}, \quad i, j=1 \text { to } 3 .
$$


Using eqns. (5), (6), and (9) while noting that the thickness is negligible compared to the other dimensions of the membrane, we get

$$
\tilde{g}_{i j}=\left(\begin{array}{ccc}
\tilde{\varrho}^{2}+\tilde{\eta}^{\prime 2} & 0 & 0 \\
0 & \tilde{\varrho}^{2} & 0 \\
0 & 0 & \lambda_{3}^{2}
\end{array}\right)
$$

and the corresponding left Cauchy-Green tensor whose eigenvalues are the squares of the principal stretches becomes

$$
\mathbf{b}=\left(G_{i j}\right)^{-1} \tilde{g}_{i j}=\left(\begin{array}{ccc}
\frac{\underline{\varrho}^{\prime 2}+\tilde{\eta}^{\prime 2}}{R_{s}^{2}} & 0 & 0 \\
0 & \frac{\tilde{\varrho}^{2}}{\left(R_{b}+R_{s} \cos \theta\right)^{2}} & 0 \\
0 & 0 & \lambda_{3}^{2}
\end{array}\right) .
$$

Now, the in-plane principal stretches $\lambda_{1}$ and $\lambda_{2}$ can be written as follows.

$$
\lambda_{1}=\frac{\sqrt{\tilde{\varrho}^{\prime 2}+\tilde{\eta}^{\prime 2}}}{R_{s}}, \quad \lambda_{2}=\frac{\tilde{\varrho}}{R_{b}+R_{s} \cos \theta} .
$$

Introducing the non-dimensional parameters $r=R_{s} / R_{b}, \varrho=\tilde{\varrho} / R_{b}$, and $\eta=\tilde{\eta} / R_{b}$,

$$
\lambda_{1}=\frac{\sqrt{\varrho^{\prime 2}+\eta^{\prime 2}}}{r}, \quad \lambda_{2}=\frac{\varrho}{1+r \cos \theta} .
$$

We note that the deformation gradient tensor in this case can be written as

$$
\mathbf{F}=\left(\begin{array}{ccc}
\lambda_{1} & 0 & 0 \\
0 & \lambda_{2} & 0 \\
0 & 0 & \lambda_{3}
\end{array}\right)
$$

\subsection{Energy considerations}

\subsubsection{Total potential energy}

Formulation presented here is based on the theory laid out in (Barham et al., 2007). The total potential energy $(E)$ of a weakly magnetizable magnetoelastic membrane can be written as follows.

$$
E=T \int_{\Omega} \rho \psi \mathrm{d} A-T \mu_{0} \int_{\Omega} \mathbf{m} \cdot \mathbf{h}_{a} \mathrm{~d} A-\int_{V_{0}}^{V_{0}+\Delta V} \tilde{P} \mathrm{~d} V,
$$

where $T$ is the undeformed membrane thickness, $\Omega$ represents the surface of the undeformed membrane, $V_{0}$ the enclosed initial volume and $\Delta V$ the change in the enclosed volume, $\rho$ is the mass density, $\psi(\mathbf{F}, \boldsymbol{\mu})$ is the free energy per unit mass defined in the formulation based on magnetization (Kankanala and Triantafyllidis, 2004; Barham et al., 2007), $\tilde{P}$ is the gauge pressure of the inflating gas, $\boldsymbol{\mu}$ is the material magnetization per unit mass, $\mathbf{m}=\rho \boldsymbol{\mu}$ is the magnetization per unit current volume, $\mu_{0}$ is the permeability of vacuum, and $\mathbf{h}_{a}$ is the applied magnetic field. Note that the formulation here is based on the assumption that the self-generated magnetic field is negligible compared to the applied field.

Now, we have the following relations (Barham et al., 2008)

$$
\frac{\partial \psi}{\partial \boldsymbol{\mu}}=\mu_{0} \mathbf{h}_{a}, \quad \rho \psi(\mathbf{F}, \boldsymbol{\mu}) \approx \widehat{W}+\frac{1}{2} C|\boldsymbol{\mu}|^{2}, \quad C=\frac{\mu_{0} \rho^{2}}{\chi}, \quad \mathbf{m}=\chi \mathbf{h}_{a},
$$

where $\chi$ is the magnetic susceptibility per unit undeformed volume, and $\widehat{W}\left(\lambda_{1}, \lambda_{2}, \lambda_{3}\right)$ is the strain energy per unit undeformed volume as explained in the next section. Note that we use the same form of energy density function here as used in other theoretical works (Dorfmann and Ogden, 2005; Barham et al., 2008) for simplicity. Similar analysis can be applied to energy density functions that are more grounded in experiments, such as those by, (Bustamante, 2010; Danas et al., 2012b; Itskov and Khim, 2016). Using the above relations in eqn. (16),

$$
E=\int_{0}^{2 \pi} \int_{0}^{2 \pi} \widehat{W} T \sqrt{G} \mathrm{~d} \theta \mathrm{d} \phi-\frac{\chi}{2} \int_{0}^{2 \pi} \int_{0}^{2 \pi} \mu_{0}\left|\mathbf{h}_{a}\right|^{2} T \sqrt{G} \mathrm{~d} \theta \mathrm{d} \phi-\int_{V_{0}}^{V_{0}+\Delta V} \tilde{P} \mathrm{~d} V
$$

where the quantity $\sqrt{G}=\left|G_{i j}\right|=R_{s}\left(R_{b}+R_{s} \cos \theta\right)$. Let the first term, elastic strain energy be denoted by $E_{\lambda}$, the second term, magnetic field energy by $E_{h}$, and the third term, pressure work by $E_{p}$. Note that the strain and magnetic field energies are calculated over the reference configuration while the pressure work is over the current configuration. 


\subsubsection{Variation in elastic strain energy}

Using the Mooney-Rivlin constitutive model for an incompressible material with material constants $C_{1}$ and $C_{2}$, the material strain energy per unit undeformed volume, $W^{*}$ is given as

$$
\begin{gathered}
W^{*}\left(I_{1}, I_{2}\right)=C_{1}\left(I_{1}-3\right)+C_{2}\left(I_{2}-3\right), \\
\text { with } \quad I_{1}=\lambda_{1}^{2}+\lambda_{2}^{2}+\lambda_{3}^{2} \quad \text { and } \quad I_{2}=\lambda_{1}^{-2}+\lambda_{2}^{-2}+\lambda_{3}^{-2},
\end{gathered}
$$

where we already used the condition for incompressibility: $\lambda_{1} \lambda_{2} \lambda_{3}=1$ or $\lambda_{3}=1 /\left(\lambda_{1} \lambda_{2}\right)$. According to this and eqns. (13) and (14),

$$
W^{*}\left(I_{1}, I_{2}\right)=\widehat{W}\left(\lambda_{1}, \lambda_{2}, \lambda_{3}\right)=\widetilde{W}\left(\varrho, \varrho^{\prime}, \tilde{\eta}, \tilde{\eta}^{\prime}\right)=W\left(\varrho, \varrho^{\prime}, \eta, \eta^{\prime}\right),
$$

and a variation in the strain energy density function will become

$$
\delta \widetilde{W}=\frac{\partial \widetilde{W}}{\partial x^{i}} \delta x^{i}, \quad \text { where }\left(x^{1}, x^{2}, x^{3}, x^{4}\right)=\left(\tilde{\varrho}, \tilde{\varrho}^{\prime}, \tilde{\eta}, \tilde{\eta}^{\prime}\right) .
$$

And the partial derivatives of $W$ can be written as

$$
\begin{aligned}
& R_{b} \frac{\partial \widetilde{W}}{\partial \tilde{\varrho}^{\prime}}=\frac{\partial W}{\partial \varrho^{\prime}}=C_{1} \frac{\partial I_{1}}{\partial \varrho^{\prime}}+C_{2} \frac{\partial I_{2}}{\partial \varrho^{\prime}}, \\
& R_{b} \frac{\partial \widetilde{W}}{\partial \varrho}=\frac{\partial W}{\partial \varrho}=C_{1} \frac{\partial I_{1}}{\partial \varrho}+C_{2} \frac{\partial I_{2}}{\partial \varrho}, \\
& R_{b} \frac{\partial \widetilde{W}}{\partial \tilde{\eta}^{\prime}}=\frac{\partial W}{\partial \eta^{\prime}}=C_{1} \frac{\partial I_{1}}{\partial \eta^{\prime}}+C_{2} \frac{\partial I_{2}}{\partial \eta^{\prime}}, \\
& R_{b} \frac{\partial \widetilde{W}}{\partial \tilde{\eta}}=\frac{\partial W}{\partial \eta}=C_{1} \frac{\partial I_{1}}{\partial \eta}+C_{2} \frac{\partial I_{2}}{\partial \eta},
\end{aligned}
$$

where using relations (19b) and (14),

$$
\begin{array}{rlrl}
\frac{\partial I_{1}}{\partial \varrho^{\prime}}=\frac{2 \varrho^{\prime}}{r^{2}}\left(1-\frac{1}{\lambda_{1}^{4} \lambda_{2}^{2}}\right), & \frac{\partial I_{2}}{\partial \varrho^{\prime}} & =\frac{2 \varrho^{\prime}}{r^{2}}\left(-\frac{1}{\lambda_{1}^{4}}+\lambda_{2}^{2}\right), \\
\frac{\partial I_{1}}{\partial \varrho}=\frac{2 \lambda_{2}}{1+r \cos \theta}\left(1-\frac{1}{\lambda_{1}^{2} \lambda_{2}^{4}}\right), & \frac{\partial I_{2}}{\partial \varrho}=\frac{2}{\lambda_{2}^{3}(1+r \cos \theta)}\left(-1+\lambda_{1}^{2} \lambda_{2}^{4}\right), \\
\frac{\partial I_{1}}{\partial \eta^{\prime}}=\frac{2 \eta^{\prime}}{r^{2}}\left(1-\frac{1}{\lambda_{1}^{4} \lambda_{2}^{2}}\right), & \frac{\partial I_{2}}{\partial \eta^{\prime}}=\frac{2 \eta^{\prime}}{r^{2}}\left(-\frac{1}{\lambda_{1}^{4}}+\lambda_{2}^{2}\right), \\
\frac{\partial I_{1}}{\partial \eta}=0, & \frac{\partial I_{2}}{\partial \eta} & =0 .
\end{array}
$$

Therefore,

$$
\begin{aligned}
\frac{\partial W}{\partial \varrho^{\prime}} & =C_{1}\left\{\frac{2 \varrho^{\prime}}{r^{2}}\left(1-\frac{1}{\lambda_{1}^{4} \lambda_{2}^{2}}\right)\right\}+C_{2}\left\{\frac{2 \varrho^{\prime}}{r^{2}}\left(-\frac{1}{\lambda_{1}^{4}}+\lambda_{2}^{2}\right)\right\} \\
& =C_{1} \frac{2 \varrho^{\prime}}{r^{2}}\left\{\left(1+\alpha \lambda_{2}^{2}\right)\left(1-\frac{1}{\lambda_{1}^{4} \lambda_{2}^{2}}\right)\right\}, \\
\frac{\partial W}{\partial \varrho} & =C_{1}\left\{\frac{2 \lambda_{2}}{1+r \cos \theta}\left(1-\frac{1}{\lambda_{1}^{2} \lambda_{2}^{4}}\right)\right\}+C_{2}\left\{\frac{2}{\lambda_{2}^{3}(1+r \cos \theta)}\left(-1+\lambda_{1}^{2} \lambda_{2}^{4}\right)\right\} \\
& =C_{1} \frac{2 \lambda_{2}}{(1+r \cos \theta)}\left\{\left(1+\alpha \lambda_{1}^{2}\right)\left(1-\frac{1}{\lambda_{1}^{2} \lambda_{2}^{4}}\right)\right\}, \\
\frac{\partial W}{\partial \eta^{\prime}} & =C_{1}\left\{\frac{2 \eta^{\prime}}{r^{2}}\left(1-\frac{1}{\lambda_{1}^{4} \lambda_{2}^{2}}\right)\right\}+C_{2}\left\{\frac{2 \eta^{\prime}}{r^{2}}\left(-\frac{1}{\lambda_{1}^{4}}+\lambda_{2}^{2}\right)\right\} \\
& =\frac{2 \eta^{\prime}}{r^{2}} C_{1}\left\{\left(1+\alpha \lambda_{2}^{2}\right)\left(1-\frac{1}{\lambda_{1}^{4} \lambda_{2}^{2}}\right)\right\}, \\
\frac{\partial W}{\partial \eta} & =0,
\end{aligned}
$$

where $\alpha=C_{2} / C_{1}$. Total strain energy of the membrane, $E_{\lambda}$ can now be written as

$$
E_{\lambda}=\int_{0}^{2 \pi} \int_{0}^{2 \pi} \widetilde{W}\left(\tilde{\varrho}, \tilde{\varrho}^{\prime}, \tilde{\eta}, \tilde{\eta}^{\prime}\right) T \sqrt{G} \mathrm{~d} \theta \mathrm{d} \phi=\int_{0}^{2 \pi} \int_{0}^{2 \pi} W\left(\varrho, \varrho^{\prime}, \eta, \eta^{\prime}\right) T \sqrt{G} \mathrm{~d} \theta \mathrm{d} \phi,
$$


and a variation in this energy, $\delta E_{\lambda}$, using eqn. (21) becomes

$$
\delta E_{\lambda}=\int_{0}^{2 \pi} \int_{0}^{2 \pi}\left(\frac{\partial \widetilde{W}}{\partial \tilde{\varrho}^{\prime}} \delta \tilde{\varrho}^{\prime}+\frac{\partial \widetilde{W}}{\partial \tilde{\varrho}} \delta \tilde{\varrho}+\frac{\partial \widetilde{W}}{\partial \tilde{\eta}^{\prime}} \delta \tilde{\eta}^{\prime}\right) T \sqrt{G} \mathrm{~d} \theta \mathrm{d} \phi
$$

We use integration by parts to convert the terms with $\delta(\cdot)^{\prime}$ to those with $\delta(\cdot)$. For example,

$$
\begin{aligned}
\int_{0}^{2 \pi} \int_{0}^{2 \pi} \frac{\partial \widetilde{W}}{\partial \widetilde{\varrho}^{\prime}} \delta \tilde{\varrho}^{\prime} T \sqrt{G} \mathrm{~d} \theta \mathrm{d} \phi & =\int_{0}^{2 \pi} \int_{0}^{2 \pi}\left\{\frac{\mathrm{d}}{\mathrm{d} \theta}\left(\frac{\partial \widetilde{W}}{\partial \tilde{\varrho}^{\prime}} \sqrt{G} \delta \tilde{\varrho}\right)-\frac{\mathrm{d}}{\mathrm{d} \theta}\left(\frac{\partial \widetilde{W}}{\partial \tilde{\varrho}^{\prime}} \sqrt{G}\right) \delta \tilde{\varrho}\right\} T \mathrm{~d} \theta \mathrm{d} \phi \\
& =-\int_{0}^{2 \pi} \int_{0}^{2 \pi}\left\{\frac{\mathrm{d}}{\mathrm{d} \theta}\left(\frac{\partial \widetilde{W}}{\partial \tilde{\varrho}^{\prime}} \sqrt{G}\right) \delta \tilde{\varrho}\right\} T \mathrm{~d} \theta \mathrm{d} \phi .
\end{aligned}
$$

Using eqns. (22) and (24), we get

$$
\delta E_{\lambda}=\int_{0}^{2 \pi} \int_{0}^{2 \pi}\left[\left\{-\frac{\mathrm{d}}{\mathrm{d} \theta}\left(\frac{\partial \widetilde{W}}{\partial \tilde{\varrho}^{\prime}} \sqrt{G}\right) T+\frac{\partial \widetilde{W}}{\partial \tilde{\varrho}} T \sqrt{G}\right\} \delta \tilde{\varrho}+\left\{-\frac{\mathrm{d}}{\mathrm{d} \theta}\left(\frac{\partial \widetilde{W}}{\partial \tilde{\eta}^{\prime}} \sqrt{G}\right) T\right\} \delta \tilde{\eta}\right] \mathrm{d} \theta \mathrm{d} \phi
$$

\subsubsection{Variation in pressure energy}

A variation in the potential energy of the inflating gas with gauge pressure $\tilde{P}$ can be written as (Steigmann, 1990, Tielking, 1975)

$$
\delta E_{p}=-\int_{0}^{2 \pi} \int_{0}^{2 \pi}(\tilde{P} \boldsymbol{n} \mathrm{d} a) \cdot \delta \boldsymbol{y}
$$

where $\mathrm{d} a=\sqrt{g} \mathrm{~d} \theta \mathrm{d} \phi$ is the area of a differential element on the deformed mid-surface $(\xi=0)$ with unit normal $\boldsymbol{n}$. Using the expressions eqn. (7),

$$
\delta E_{p}=\int_{0}^{2 \pi} \int_{0}^{2 \pi} \tilde{P} \epsilon_{i j k} y_{, 1}^{j} y_{, 2}^{k} \delta y^{i} \mathrm{~d} \theta \mathrm{d} \phi
$$

Now the variations in $y^{i}$ can be written, from eqn. (5b), as

$$
\delta y^{1}=\delta \tilde{\varrho} \cos \phi, \quad \delta y^{2}=\delta \varrho \sin \phi, \quad \delta y^{3}=\delta \tilde{\eta} .
$$

Therefore,

$$
\begin{aligned}
\delta E_{p} & =-\int_{0}^{2 \pi} \int_{0}^{2 \pi} \tilde{P}\left\{\delta \varrho \tilde{c o s} \phi\left(\tilde{\varrho} \tilde{\eta}^{\prime} \cos \phi\right)+\delta \varrho \sin \phi\left(\tilde{\varrho} \tilde{\eta}^{\prime} \sin \phi\right)+\delta \tilde{\eta}\left(-\tilde{\varrho} \tilde{\varrho}^{\prime}\right)\right\} \mathrm{d} \theta \mathrm{d} \phi \\
& =-\int_{0}^{2 \pi} \int_{0}^{2 \pi} \tilde{P}\left(\tilde{\varrho} \tilde{\eta}^{\prime} \delta \tilde{\varrho}-\tilde{\varrho} \tilde{\varrho}^{\prime} \delta \tilde{\eta}\right) \mathrm{d} \theta \mathrm{d} \phi \\
& =\int_{0}^{2 \pi} \int_{0}^{2 \pi}\left[\left(-\tilde{P} \tilde{\varrho} \tilde{\eta}^{\prime}\right) \delta \tilde{\varrho}+\frac{\mathrm{d}}{\mathrm{d} \theta}\left(\frac{1}{2} \tilde{P} \tilde{\varrho}^{2}\right) \delta \tilde{\eta}\right] \mathrm{d} \theta \mathrm{d} \phi .
\end{aligned}
$$

\subsubsection{Variation in energy of the magnetic field}

We generate a magnetic field in the deforming membrane by placing a current carrying loop on a circle of radius $\tilde{a}$ on the $Y^{1}-Y^{2}$ plane. This magnetic field strength $\mathbf{h}_{a}$ can be obtained as follows.

$$
\mathrm{d} \mathbf{h}_{a}=\frac{I}{4 \pi} \frac{\mathrm{d} \boldsymbol{l} \times \boldsymbol{s}}{|\boldsymbol{s}|^{3}},
$$

where $I$ is the current. A differential length element on the wire, $\mathrm{d} \boldsymbol{l}$ at an angle $\phi_{i}$, and the position of a point on the mid-surface of the toroid from $\mathrm{d} \boldsymbol{l}, \boldsymbol{s}$ (see Figure 2) are as follows.

$$
\begin{gathered}
\mathrm{d} \boldsymbol{l}=-\mathrm{d} l \sin \phi_{i} \boldsymbol{e}_{1}+\mathrm{d} l \cos \phi_{i} \boldsymbol{e}_{2}, \quad \mathrm{~d} l=\tilde{a} \mathrm{~d} \phi_{i}, \\
\boldsymbol{s}=\left(\tilde{\varrho} \cos \phi-\tilde{a} \cos \phi_{i}\right) \boldsymbol{e}_{1}+\left(\varrho \tilde{\varrho} \sin \phi-\tilde{a} \sin \phi_{i}\right) \boldsymbol{e}_{2}+\tilde{\eta} \boldsymbol{e}_{3},
\end{gathered}
$$

where $\tilde{a}$ stands for the position of the current carrying wire and $\left\{\boldsymbol{e}_{i}\right\}$ is the set of orthonormal basis vectors. We have

$$
\mathrm{d} \boldsymbol{l} \times \boldsymbol{s}=\mathrm{d} \phi_{i}\left[\tilde{\eta} \tilde{a} \cos \phi_{i} \boldsymbol{e}_{1}+\tilde{\eta} \tilde{a} \sin \phi_{i} \boldsymbol{e}_{2}+\left(\tilde{a}^{2}-\tilde{\varrho} \tilde{a} \cos \left(\phi_{i}-\phi\right)\right) \boldsymbol{e}_{3}\right]
$$




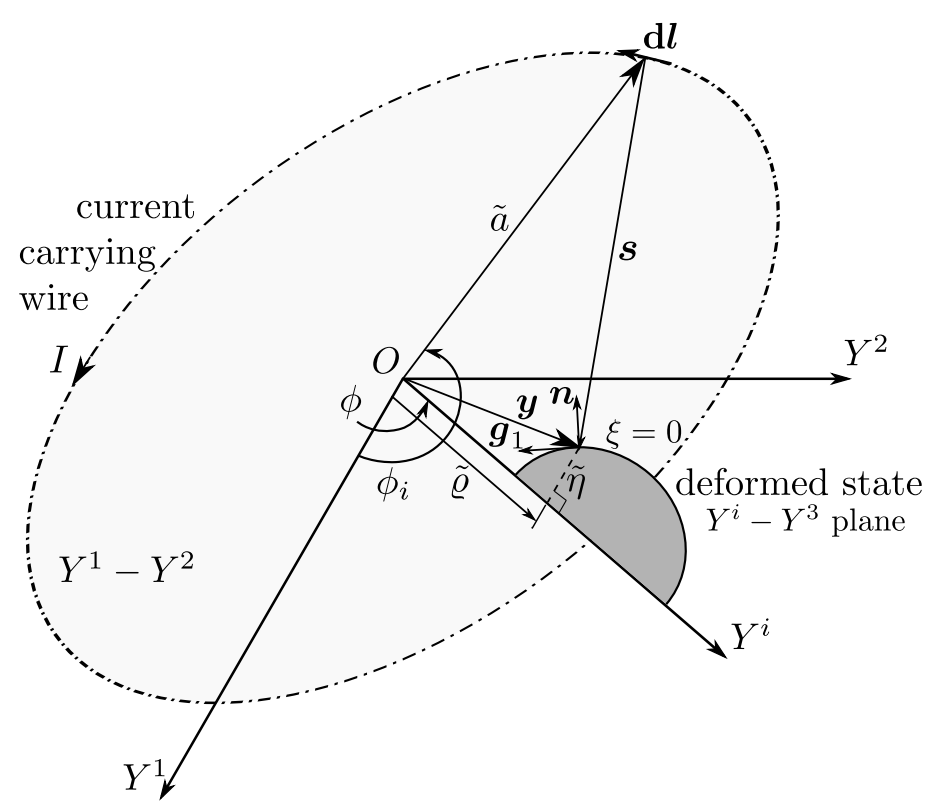

Figure 2: Situation at an arbitrary time instant. The current carrying wire is placed along a circle of radius $\tilde{a}$ on $Y^{1}-Y^{2}$ plane and the deformed cross-section of the membrane is on $Y^{i}-Y^{3}$ plane. Only the mid-surface of the deformed state is shown.

Now, the magnetic field strength $\mathbf{h}_{a}$ can be written as follows.

$$
\mathbf{h}_{a}=\frac{I}{4 \pi} \int_{0}^{2 \pi} \frac{\boldsymbol{e}_{1}\left(\tilde{\eta} \tilde{a} \cos \phi_{i}\right)+\boldsymbol{e}_{2}\left(\tilde{\eta} \tilde{a} \sin \phi_{i}\right)+\boldsymbol{e}_{3}\left(\tilde{a}^{2}-\tilde{\varrho} \tilde{a} \cos \left(\phi_{i}-\phi\right)\right)}{\left(\varrho^{2}+\tilde{\eta}^{2}+\tilde{a}^{2}-2 \tilde{\varrho} \tilde{a} \cos \left(\phi_{i}-\phi\right)\right)^{3 / 2}} \mathrm{~d} \phi_{i},
$$

Expressing in terms of the non-dimensional quantities $(\varrho, \eta, a)=(\tilde{\varrho}, \tilde{\eta}, \tilde{a}) / R_{b}$,

$$
\mathbf{h}_{a}=\frac{I}{4 \pi R_{b}} \int_{0}^{2 \pi} \frac{\boldsymbol{e}_{1}\left(\eta a \cos \phi_{i}\right)+\boldsymbol{e}_{2}\left(\eta a \sin \phi_{i}\right)+\boldsymbol{e}_{3}\left(a^{2}-\varrho a \cos \left(\phi_{i}-\phi\right)\right)}{\left(\varrho^{2}+\eta^{2}+a^{2}-2 \varrho a \cos \left(\phi_{i}-\phi\right)\right)^{3 / 2}} \mathrm{~d} \phi_{i}
$$

This expression is same as that derived by (Grivich and Jackson, 2000, eqn. 15) as a limiting case of magnetic field due to current-carrying polygons.

In the current study, position of the current carrying loop $(\tilde{a})$ for a given position of the outer edge $(\varrho(0))$ is taken to be coinciding with the centre of the deformed cross-section if it were always circular i.e., centre of the parametrically deformed cross-section (explained in Section 3).

For our analysis later, it is convenient to express the magnetic field intensity in the curvilinear coordinates associated with the deformed configuration. From the expression (6), the unit bases vectors along the coordinates $(\widehat{\theta}, \widehat{\phi}, \widehat{\xi})$, with the hat $\widehat{(\cdot)}$ representing the corresponding unit vector, can be written as follows.

$$
\begin{gathered}
\widehat{\boldsymbol{g}}_{i}=T_{i j} \boldsymbol{e}_{j}, \\
\text { where } T_{11}=\frac{\varrho^{\prime} \cos \phi_{i}}{\sqrt{\varrho^{\prime 2}+\eta^{\prime 2}}}, T_{12}=\frac{\varrho^{\prime} \sin \phi_{i}}{\sqrt{\varrho^{\prime 2}+\eta^{\prime 2}}}, T_{13}=\frac{\eta^{\prime}}{\sqrt{\varrho^{\prime 2}+\eta^{\prime 2}}}, \\
T_{21}=-\sin \phi_{i}, T_{22}=\cos \phi_{i}, T_{23}=0, T_{31}=-n^{1}, T_{32}=-n^{2}, T_{33}=-n^{3},
\end{gathered}
$$

since $\widehat{\boldsymbol{g}}_{3}=-\boldsymbol{n}$ from the definition (7). Similarly, inverting the above relation, the Cartesian bases vectors can be written as

$$
\begin{gathered}
\boldsymbol{e}_{i}=\tilde{T}_{i j} \widehat{\boldsymbol{g}}_{j}, \\
\text { where } \quad \tilde{T}_{11}=\frac{\varrho^{\prime} \cos \phi_{i}}{\sqrt{\varrho^{\prime 2}+\eta^{\prime 2}}}, \tilde{T}_{12}=-\sin \phi_{i}, \tilde{T}_{13}=-\frac{\eta^{\prime} \cos \phi_{i}}{\sqrt{\varrho^{\prime 2}+\eta^{\prime 2}}}, \tilde{T}_{21}=\frac{\varrho^{\prime} \sin \phi_{i}}{\sqrt{\varrho^{\prime 2}+\eta^{\prime 2}}}, \\
\tilde{T}_{22}=\cos \phi_{i}, \tilde{T}_{23}=-\frac{\eta^{\prime} \sin \phi_{i}}{\sqrt{\varrho^{\prime 2}+\eta^{\prime 2}}}, \tilde{T}_{31}=\frac{\eta^{\prime}}{\sqrt{\varrho^{\prime 2}+\eta^{\prime 2}}}, \tilde{T}_{32}=0, \tilde{T}_{33}=\frac{\varrho^{\prime}}{\sqrt{\varrho^{\prime 2}+\eta^{\prime 2}}} .
\end{gathered}
$$


Using the above relations in the expression for magnetic field intensity (38),

$$
\begin{aligned}
\mathbf{h}_{a}=\frac{I}{4 \pi R_{b}} & \int_{0}^{2 \pi} \widehat{\boldsymbol{g}}_{1} \frac{\varrho^{\prime} \eta a+\eta^{\prime}\left(a^{2}-\varrho a \cos \left(\phi_{i}-\phi\right)\right)}{\sqrt{\varrho^{\prime 2}+\eta^{\prime 2}}\left(\varrho^{2}+\eta^{2}+a^{2}-2 \varrho a \cos \left(\phi_{i}-\phi\right)\right)^{3 / 2}} \mathrm{~d} \phi_{i} \\
& +\frac{I}{4 \pi R_{b}} \int_{0}^{2 \pi} \widehat{\boldsymbol{g}}_{3} \frac{-\eta^{\prime} \eta a+\varrho^{\prime}\left(a^{2}-\varrho a \cos \left(\phi_{i}-\phi\right)\right)}{\sqrt{\varrho^{\prime 2}+\eta^{\prime 2}}\left(\varrho^{2}+\eta^{2}+a^{2}-2 \varrho a \cos \left(\phi_{i}-\phi\right)\right)^{3 / 2}} \mathrm{~d} \phi_{i},
\end{aligned}
$$

It can be seen that the above formulation confirms with the physics of the situation: magnetic field intensity vanishes along the coordinate $\phi$. Also notice that both the forms (38) and (41) lead to the following absolute value

$$
\left|\mathbf{h}_{a}\right|^{2}=\frac{I^{2}}{16 \pi^{2} R_{b}^{2}} \int_{0}^{2 \pi} \frac{\eta^{2} a^{2}+\left(a^{2}-\varrho a \cos \left(\phi_{i}-\phi\right)\right)^{2}}{\left(\varrho^{2}+\eta^{2}+a^{2}-2 \varrho a \cos \left(\phi_{i}-\phi\right)\right)^{3}} \mathrm{~d} \phi_{i} .
$$

Let the expressions (38) and (41) be written shortly as

$$
\mathbf{h}_{a}=\frac{I}{4 \pi R_{b}}\left(\boldsymbol{e}_{1}\left(h_{a}\right)_{1}+\boldsymbol{e}_{2}\left(h_{a}\right)_{2}+\boldsymbol{e}_{3}\left(h_{a}\right)_{3}\right)=\frac{I}{4 \pi R_{b}}\left(\widehat{\boldsymbol{g}}_{1}\left(h_{a}\right)_{\theta}+\widehat{\boldsymbol{g}}_{3}\left(h_{a}\right)_{\xi}\right) .
$$

Now, from eqn. (18), magnetic energy $E_{h}$ can be written as

$$
E_{h}=-\frac{\chi}{2} \int_{0}^{2 \pi} \int_{0}^{2 \pi} \mathcal{H} T \sqrt{G} \mathrm{~d} \theta \mathrm{d} \phi
$$

where the parameter $\mathcal{H}$ introduced to represent the magnetic field energy density is

$$
\mathcal{H}=\mu_{0}\left|\mathbf{h}_{a}\right|^{2}=\frac{\mu_{0} I^{2}}{16 \pi^{2} R_{b}^{2}}\left(\left(h_{a}\right)_{1}^{2}+\left(h_{a}\right)_{2}^{2}+\left(h_{a}\right)_{3}^{2}\right)=C_{1} \mathcal{M}\left(\left(h_{a}\right)_{1}^{2}+\left(h_{a}\right)_{2}^{2}+\left(h_{a}\right)_{3}^{2}\right),
$$

where $\mathcal{M}$ as shown below is a non-dimensional parameter introduced to represent the magnetic field energy density

$$
\mathcal{M}=\frac{\mu_{0} I^{2}}{16 \pi^{2} R_{b}^{2} C_{1}} .
$$

To get an idea of the current required, let $C_{1} \approx 10^{5} \mathrm{~N} / \mathrm{m}^{2}, R_{b} \approx 10^{-3} \mathrm{~m}$, and $\mathcal{M}=1 \times 10^{-04}$. Then from the above definition, $I \approx 35.45$ A.

Now, a variation in the magnetic energy can be written as

$$
\begin{gathered}
\delta E_{h}=-\frac{\chi}{2} \int_{0}^{2 \pi} \int_{0}^{2 \pi}\left(\frac{\partial \mathcal{H}}{\partial \tilde{\varrho}} \delta \tilde{\varrho}+\frac{\partial \mathcal{H}}{\partial \tilde{\eta}} \delta \tilde{\eta}\right) T \sqrt{G} \mathrm{~d} \theta \mathrm{d} \phi \\
\text { or } \delta E_{h}=\int_{0}^{2 \pi} \int_{0}^{2 \pi}\left[\left(-\frac{\chi}{2} \frac{\partial \mathcal{H}}{\partial \varrho} T \sqrt{G}\right) \delta \tilde{\varrho}+\left(-\frac{\chi}{2} \frac{\partial \mathcal{H}}{\partial \tilde{\eta}} T \sqrt{G}\right) \delta \tilde{\eta}\right] \mathrm{d} \theta \mathrm{d} \phi .
\end{gathered}
$$

\subsection{Governing equations}

From the principle of minimum potential energy, equilibrium is achieved when upon any virtual displacement

$$
\delta E=\delta E_{\lambda}+\delta E_{h}+\delta E_{p}=0
$$

Using eqns. (28), (32), and (48),

$$
\begin{aligned}
\int_{0}^{2 \pi} \int_{0}^{2 \pi} & {\left[\left\{-\frac{\mathrm{d}}{\mathrm{d} \theta}\left(\frac{\partial \widetilde{W}}{\partial \tilde{\varrho}^{\prime}} \sqrt{G}\right) T+\frac{\partial \widetilde{W}}{\partial \tilde{\varrho}} T \sqrt{G}-\frac{\chi}{2} \frac{\partial \mathcal{H}}{\partial \tilde{\varrho}} T \sqrt{G}-\tilde{P} \tilde{\varrho} \tilde{\eta}^{\prime}\right\} \delta \tilde{\varrho}\right.} \\
+ & \left.\left\{-\frac{\mathrm{d}}{\mathrm{d} \theta}\left(\frac{\partial \widetilde{W}}{\partial \tilde{\eta}^{\prime}} \sqrt{G}\right) T-\frac{\chi}{2} \frac{\partial \mathcal{H}}{\partial \tilde{\eta}} T \sqrt{G}+\frac{\mathrm{d}}{\mathrm{d} \theta}\left(\frac{1}{2} \tilde{P} \tilde{\varrho}^{2}\right)\right\} \delta \tilde{\eta}\right] \mathrm{d} \theta \mathrm{d} \phi=0 .
\end{aligned}
$$

Separating the coefficients of $\delta \varrho \tilde{~ a n d} \delta \tilde{\eta}$,

$$
\begin{gathered}
\frac{\mathrm{d}}{\mathrm{d} \theta}\left(\frac{\partial \widetilde{W}}{\partial \tilde{\varrho}^{\prime}} \sqrt{G}\right)-\frac{\partial \widetilde{W}}{\partial \tilde{\varrho}} \sqrt{G}+\frac{1}{2} \chi \frac{\partial \mathcal{H}}{\partial \tilde{\varrho}} \sqrt{G}+\frac{1}{T} \tilde{P} \tilde{\varrho} \tilde{\eta}^{\prime}=0, \\
\frac{\mathrm{d}}{\mathrm{d} \theta}\left(\frac{\partial \widetilde{W}}{\partial \tilde{\eta}^{\prime}} \sqrt{G}-\frac{1}{2 T} \tilde{P} \tilde{\varrho}^{2}\right)+\frac{1}{2} \chi \frac{\partial \mathcal{H}}{\partial \tilde{\eta}} \sqrt{G}=0 .
\end{gathered}
$$


Using (22), (24), and (45),

$$
\begin{aligned}
& \frac{\mathrm{d}}{\mathrm{d} \theta}\{\left.\frac{C_{1}}{R_{b}} \frac{2 \varrho^{\prime}}{r^{2}}\left(1+\alpha \lambda_{2}^{2}\right)\left(1-\frac{1}{\lambda_{1}^{4} \lambda_{2}^{2}}\right) R_{b}^{2} r(1+r \cos \theta)\right\} \\
&-\frac{C_{1}}{R_{b}} \frac{2 \lambda_{2}}{1+r \cos \theta}\left(1+\alpha \lambda_{1}^{2}\right)\left(1-\frac{1}{\lambda_{1}^{2} \lambda_{2}^{4}}\right) R_{b}^{2} r(1+r \cos \theta) \\
&+\frac{\chi}{2 R_{b}} C_{1} \mathcal{M}\left[\frac{\partial}{\partial \varrho}\left(\left(h_{a}\right)_{1}^{2}+\left(h_{a}\right)_{2}^{2}+\left(h_{a}\right)_{3}^{2}\right)\right] R_{b}^{2} r(1+r \cos \theta)+\frac{1}{T} \tilde{P} \varrho \eta^{\prime} R_{b}^{2}=0, \\
& \frac{\mathrm{d}}{\mathrm{d} \theta}\left\{\frac{C_{1}}{R_{b}} \frac{2 \eta^{\prime}}{r^{2}}\left(1+\alpha \lambda_{2}^{2}\right)\left(1-\frac{1}{\lambda_{1}^{4} \lambda_{2}^{2}}\right) R_{b}^{2} r(1+r \cos \theta)-\frac{1}{2 T} \tilde{P} \varrho^{2} R_{b}^{2}\right\} \\
& \quad+\frac{\chi}{2 R_{b}} C_{1} \mathcal{M}\left[\frac{\partial}{\partial \eta}\left(\left(h_{a}\right)_{1}^{2}+\left(h_{a}\right)_{2}^{2}+\left(h_{a}\right)_{3}^{2}\right)\right] R_{b}^{2} r(1+r \cos \theta)=0 .
\end{aligned}
$$

Introducing the non-dimensional gauge pressure $P=\tilde{P} R_{b} / C_{1} T$, the governing equations can be written as

$$
\begin{aligned}
\frac{\mathrm{d}}{\mathrm{d} \theta}\left\{\frac{\varrho^{\prime}}{r}\left(1+\alpha \lambda_{2}^{2}\right)(1\right. & \left.\left.-\frac{1}{\lambda_{1}^{4} \lambda_{2}^{2}}\right)(1+r \cos \theta)\right\}-r \lambda_{2}\left(1+\alpha \lambda_{1}^{2}\right)\left(1-\frac{1}{\lambda_{1}^{2} \lambda_{2}^{4}}\right) \\
+\frac{1}{4} \chi \mathcal{M}\left[\frac{\partial}{\partial \varrho}\left(\left(h_{a}\right)_{1}^{2}+\left(h_{a}\right)_{2}^{2}+\left(h_{a}\right)_{3}^{2}\right)\right] r(1+r \cos \theta)+\frac{1}{2} P \varrho \eta^{\prime}=0, & \\
\frac{\mathrm{d}}{\mathrm{d} \theta}\left\{\frac{\eta^{\prime}}{r}\left(1+\alpha \lambda_{2}^{2}\right)\right. & \left.\left(1-\frac{1}{\lambda_{1}^{4} \lambda_{2}^{2}}\right)(1+r \cos \theta)-\frac{1}{4} P \varrho^{2}\right\} \\
& +\frac{1}{4} \chi \mathcal{M}\left[\frac{\partial}{\partial \eta}\left(\left(h_{a}\right)_{1}^{2}+\left(h_{a}\right)_{2}^{2}+\left(h_{a}\right)_{3}^{2}\right)\right] r(1+r \cos \theta)=0 .
\end{aligned}
$$

\subsection{Solution Procedure}

The governing equations (55) and (56) are to be solved for the non-dimensional quantities $\varrho, \varrho^{\prime}, \eta$, and $\eta^{\prime}$ for the loading parameters $\mathcal{M}$ and $P$. The boundary conditions based on the symmetry with respect to $Y^{1}-Y^{2}$ plane are (see Figure 1)

$$
\varrho^{\prime}(0)=0, \varrho^{\prime}(\pi)=0, \eta(0)=0, \eta(\pi)=0 .
$$

Since the pressure-stretch curve is not monotonic, to trace it past the limit-point, we treat pressure as an unknown and initiate the numerical solution procedure with a value of $\varrho(0)$, distance of the outer edge of the membrane from the origin as an input. Now the coupled second order ordinary differential equations (55) and (56) can be brought to the following form.

$$
\mathbf{A} \mathbf{X}^{\prime}=\mathbf{E}
$$

with $\mathbf{X}=(u, v, w, x)^{t}, \mathbf{X}^{\prime}=\left(u^{\prime}, v^{\prime}, w^{\prime}, x^{\prime}\right)^{t},(\cdot)^{t}$ representing transposition,

$$
u=\varrho, v=\varrho^{\prime}, w=\eta, x=\eta^{\prime},
$$

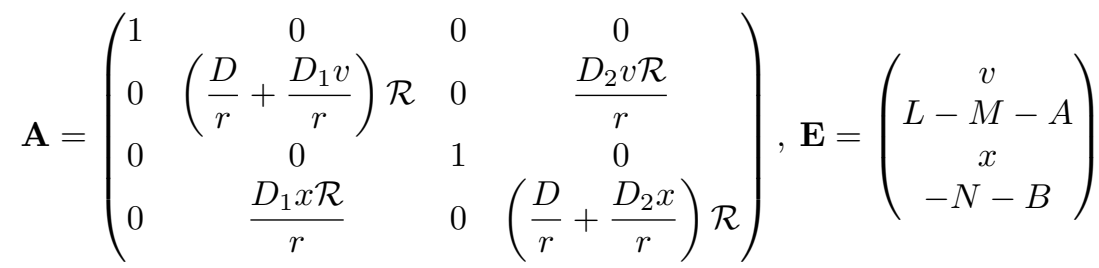

$$
\begin{aligned}
& A=\frac{D_{3} v \mathcal{R}}{r}-D v \sin \theta, B=\frac{D_{3} x \mathcal{R}}{r}-D x \sin \theta-\frac{1}{2} P u v, \\
& \text { with } \mathcal{R}=(1+r \cos \theta), \quad D=\mathcal{C} U, D_{1}=\mathcal{C} U_{1}, \quad D_{2}=\mathcal{C} U_{2}, \quad D_{3}=\mathcal{C}_{1} U+\mathcal{C} U_{3} \text {, } \\
& \mathcal{C}=1+\alpha \frac{u^{2}}{\mathcal{R}^{2}}, \quad U=1-\frac{r^{4} \mathcal{R}^{2}}{z^{4} u^{2}}, \mathcal{C}_{1}=\alpha\left(\frac{2 u v}{\mathcal{R}^{2}}+\frac{2 r \sin \theta}{\mathcal{R}^{3}} u^{2}\right), \\
& U_{1}=\frac{4 \mathcal{R}^{2} r^{4} v}{z^{6} u^{2}}, U_{2}=\frac{4 \mathcal{R}^{2} r^{4} x}{z^{6} u^{2}}, U_{3}=\frac{2 \mathcal{R}^{2} r^{4} v}{z^{4} u^{3}}+\frac{2 \mathcal{R} r^{5} \sin \theta}{z^{4} u^{2}}, z=\sqrt{v^{2}+x^{2}}, \\
& L=r \frac{u}{\mathcal{R}}\left(1+\alpha \frac{z^{2}}{r^{2}}\right)\left(1-\frac{r^{2} \mathcal{R}^{4}}{z^{2} u^{4}}\right)-\frac{1}{2} P u x, \quad M=\frac{1}{4} \chi \mathcal{M}\left[\frac{\partial}{\partial \varrho}\left(\left(h_{a}\right)_{1}^{2}+\left(h_{a}\right)_{2}^{2}+\left(h_{a}\right)_{3}^{2}\right)\right] r \mathcal{R}, \\
& N=\frac{1}{4} \chi \mathcal{M}\left[\frac{\partial}{\partial \eta}\left(\left(h_{a}\right)_{1}^{2}+\left(h_{a}\right)_{2}^{2}+\left(h_{a}\right)_{3}^{2}\right)\right] r \mathcal{R},
\end{aligned}
$$


with the boundary conditions determined by the symmetry of the cross-section

$$
v(0)=0, v(\pi)=0, w(0)=0, w(\pi)=0 .
$$

The above boundary value problem is converted into an initial value problem using shooting method and the resultant is solved using a fourth-order Runge-Kutta method (via ode45 in MATLAB ${ }^{\circledR}$ ). For a given value of the location of the outer edge $\varrho(0)$, we start with a guess pair $\left(\eta^{\prime}(0), P\right)$, and apply shooting method to obtain the pair $\left(\varrho^{\prime}(\pi), \eta(\pi)\right)$ whose value should ideally be $(0,0)$. The correct pair $\left(\eta^{\prime}(0), P\right)$ that makes the quantity $\sqrt{\varrho^{\prime}(\pi)^{2}+\eta(\pi)^{2}}$ sufficiently small $\left(\mathcal{O}\left(10^{-13}\right)\right)$ is obtained using Nelder-Meads optimization technique (via fminsearch in MATLAB ${ }^{\circledR}$ ). For this correct pair, the Runge-Kutta method gives the values of $(u, v, w, x)$ or equivalently $\left(\varrho, \varrho^{\prime}, \eta, \eta^{\prime}\right)$ over the domain $\theta \in[0, \pi]$.

The integrals involved in the expressions related to magnetic field intensity: (38), (41), (42) are evaluated numerically using trapezoidal method (via trapz in MATLAB ${ }^{\circledR}$ ) with a sufficiently fine mesh ensuring the convergence of the integral values.

\subsection{Second Variation}

Second variation of the potential energy functional (18) can be written as (Gelfand and Fomin, 2000, Ch. 5)

$$
\delta^{2} E=\int_{0}^{2 \pi} \int_{0}^{2 \pi}\left(\left\langle\mathbf{P} \boldsymbol{\Delta}^{\prime}, \boldsymbol{\Delta}^{\prime}\right\rangle+\langle\mathbf{Q} \boldsymbol{\Delta}, \boldsymbol{\Delta}\rangle\right) \mathrm{d} \theta \mathrm{d} \phi
$$

where $\langle$,$\rangle represents inner product and the variation vector \Delta$ is given as

$$
\Delta=\left\{\begin{array}{l}
\delta \varrho \\
\delta \eta
\end{array}\right\}
$$

The symmetric matrices $\mathbf{P}$ and $\mathbf{Q}$, taking advantage of the fact that the integrand in (18) is independent of the circumferential coordinate $\phi$, are as follows

$$
\mathbf{P}=\frac{1}{2}\left(\begin{array}{cc}
\mathcal{F}_{\varrho^{\prime} \varrho^{\prime}} & \mathcal{F}_{\varrho^{\prime} \eta^{\prime}} \\
\mathcal{F}_{\eta^{\prime} \varrho^{\prime}} & \mathcal{F}_{\eta^{\prime} \eta^{\prime}}
\end{array}\right), \quad \mathbf{Q}=\frac{1}{2}\left(\begin{array}{cc}
\mathcal{F}_{\varrho \varrho} & \mathcal{F}_{\varrho \eta} \\
\mathcal{F}_{\eta \varrho} & \mathcal{F}_{\eta \eta}
\end{array}\right)-\frac{1}{2} \frac{\mathrm{d}}{\mathrm{d} \theta}\left(\begin{array}{cc}
\mathcal{F}_{\varrho \varrho^{\prime}} & \mathcal{F}_{\varrho \eta^{\prime}} \\
\mathcal{F}_{\eta \varrho^{\prime}} & \mathcal{F}_{\eta \eta^{\prime}}
\end{array}\right),
$$

with

$$
\mathcal{F}=\widehat{W} T \sqrt{G}-\frac{\chi}{2} \mu_{0}\left|\mathbf{h}_{a}\right|^{2} T \sqrt{G}-\frac{1}{2} \tilde{P} \tilde{\rho}^{2} \tilde{\eta}^{\prime}
$$

being the integrand in eqn. (18) and for example, $\mathcal{F}_{\varrho \eta^{\prime}}=\partial^{2} \mathcal{F} / \partial \varrho \partial \eta^{\prime}$.

A necessary condition for the equilibrium state obtained from solution of the governing equations (55) and (56) to be a minimizer of the energy functional (18) is that the matrix $\mathbf{P}$ be positive definite for all $\theta \in[0,2 \pi]$. A simple but rather restrictive sufficient condition for minimization (Gelfand and Fomin, 2000, Ch. 5) is that the solution to the following differential equation exists and is invertible

$$
-\frac{\mathrm{d}}{\mathrm{d} \theta}\left(\mathbf{P} \mathbf{U}^{\prime}\right)+\mathbf{Q U}=0, \quad \mathbf{U}(0)=\left(\begin{array}{ll}
0 & 0 \\
0 & 0
\end{array}\right), \quad \mathbf{U}^{\prime}(0)=\left(\begin{array}{ll}
1 & 0 \\
0 & 1
\end{array}\right),
$$

for all $\theta \in[0,2 \pi]$. Although the sufficient condition is strong, it works well for all cases except a few where only the necessary condition is satisfied.

\section{Parametric deformation}

This section deals with a special case of deformation in which the cross-section of toroid is assumed to remain circular as demonstrated in Figure 3. We choose two parameters to characterise the geometry at any stage. As shown, $\tilde{a}=\tilde{\varrho}(\theta=\pi / 2)$ represents the radius of the centre-line of the torus while $\tilde{b}$ stands for the radius of the circular cross-section.

From the geometry, we have the following relations

$$
\begin{array}{ll}
\tilde{\varrho}=\tilde{a}+\tilde{b} \cos \theta, & \tilde{\eta}=\tilde{b} \sin \theta, \\
\tilde{\varrho}^{\prime}=-\tilde{b} \sin \theta, & \tilde{\eta}^{\prime}=\tilde{b} \cos \theta .
\end{array}
$$

We define the following non-dimensional quantities for further use.

$$
\varrho=\frac{\tilde{\varrho}}{R_{b}}, \eta=\frac{\tilde{\eta}}{R_{b}}, a=\frac{\tilde{a}}{R_{b}}, \text { and } b=\frac{\tilde{b}}{R_{b}} .
$$




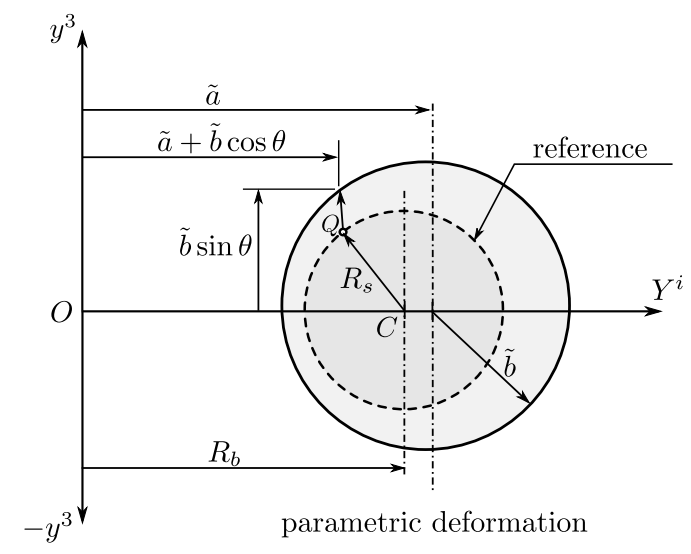

Figure 3: Toroidal membrane after parametric deformation illustrated through a point $Q$ on $Y^{i}-Y^{3}$ plane. Centre of the parametrically deformed circular cross-section is at a distance $\tilde{a}$ from the origin $O$. The reference configuration and the point $Q$ are as shown in Figure 1.

We can see that this choice of parameters satisfies the boundary conditions

$$
\begin{array}{ccc}
\tilde{\varrho}^{\prime}(0)=-\tilde{b} \sin (0)=0, & \tilde{\varrho}^{\prime}(\pi)=-\tilde{b} \sin (\pi)=0 . \\
\tilde{\eta}(0)=\tilde{b} \sin (0)=0, & \tilde{\eta}(\pi)=\tilde{b} \sin (\pi)=0 .
\end{array}
$$

Now, the principal stretches can be written from eqns. (4), (13) and (14) as

$$
\lambda_{1}=\frac{\tilde{b}}{R_{s}}=\frac{b}{r}, \quad \lambda_{2}=\frac{\tilde{a}+\tilde{b} \cos \theta}{R_{b}+R_{s} \cos \theta}=\frac{a+b \cos \theta}{1+r \cos \theta}, \quad \lambda_{3}=\frac{t}{T} .
$$

We use the same concepts discussed earlier to arrive at the governing equations in terms of $\delta \tilde{a}$ and $\delta \tilde{b}$. The strain energy density can also be written from eqn. (20) as

$$
\widehat{W}\left(\lambda_{1}, \lambda_{2}, \lambda_{3}\right)=\widehat{U}^{*}(\tilde{a}, \tilde{b})=\widehat{U}(a, b) .
$$

Therefore, a variation in strain energy density can be written as

$$
\delta E_{\lambda}=\int_{0}^{2 \pi} \int_{0}^{2 \pi} \frac{1}{R_{b}}\left[\left(\frac{\partial \widehat{U}}{\partial a} T \sqrt{G}\right) \delta \tilde{a}+\left(\frac{\partial \widehat{U}}{\partial b} T \sqrt{G}\right) \delta \tilde{b}\right] \mathrm{d} \theta \mathrm{d} \phi,
$$

where, $\sqrt{G}=R_{b}^{2} r(1+r \cos \theta)$. Using chain rule for partial differentials and relations (24),

$$
\begin{aligned}
\frac{\partial \widehat{U}}{\partial a} & =\frac{\partial W}{\partial \varrho} \frac{\partial \varrho}{\partial a} \\
& =2 C_{1}\left(\frac{\lambda_{2}}{1+r \cos \theta}\right)\left(1+\alpha \lambda_{1}^{2}\right)\left(1-\frac{1}{\lambda_{1}^{2} \lambda_{2}^{4}}\right), \\
\frac{\partial \widehat{U}}{\partial b} & =\frac{\partial W}{\partial \varrho} \frac{\partial \varrho}{\partial b}+\frac{\partial W}{\partial \varrho^{\prime}} \frac{\partial \varrho^{\prime}}{\partial b}+\frac{\partial W}{\partial \eta} \frac{\partial \eta}{\partial b}+\frac{\partial W}{\partial \eta^{\prime}} \frac{\partial \eta^{\prime}}{\partial b} \\
& =2 C_{1}\left(\frac{\lambda_{1}}{r}\right)\left(1+\alpha \lambda_{2}^{2}\right)\left(1-\frac{1}{\lambda_{1}^{4} \lambda_{2}^{2}}\right)+2 C_{1}\left(\frac{\lambda_{2} \cos \theta}{1+r \cos \theta}\right)\left(1+\alpha \lambda_{1}^{2}\right)\left(1-\frac{1}{\lambda_{1}^{2} \lambda_{2}^{4}}\right) .
\end{aligned}
$$

Similarly, variation in magnetic field energy can be written as (see eqn. (48))

$$
\delta E_{h}=\int_{0}^{2 \pi} \int_{0}^{2 \pi} \frac{-\chi}{2 R_{b}}\left[\left(\frac{\partial \mathcal{H}}{\partial a} T \sqrt{G}\right) \delta \tilde{a}+\left(\frac{\partial \mathcal{H}}{\partial b} T \sqrt{G}\right) \delta \tilde{b}\right] \mathrm{d} \theta \mathrm{d} \phi,
$$

where, from (45),

$$
\begin{aligned}
\frac{\partial \mathcal{H}}{\partial a} & =C_{1} \mathcal{M} \frac{\partial\left(\left(h_{a}\right)_{1}^{2}+\left(h_{a}\right)_{2}^{2}+\left(h_{a}\right)_{3}^{2}\right)}{\partial a}, \\
\frac{\partial \mathcal{H}}{\partial b} & =C_{1} \mathcal{M} \frac{\partial\left(\left(h_{a}\right)_{1}^{2}+\left(h_{a}\right)_{2}^{2}+\left(h_{a}\right)_{3}^{2}\right)}{\partial b},
\end{aligned}
$$


where, using the relations (65), and eqns. (38), and (43),

$$
\begin{aligned}
\left(h_{a}\right)_{1} & =\int_{0}^{2 \pi} \frac{a b \sin \theta \cos \phi_{i}}{\left\{b^{2}+2 a(a+b \cos \theta)\left(1-\cos \left(\phi_{i}-\phi\right)\right)\right\}^{3 / 2}} \mathrm{~d} \phi_{i}, \\
\left(h_{a}\right)_{2} & =\int_{0}^{2 \pi} \frac{a b \sin \theta \sin \phi_{i}}{\left\{b^{2}+2 a(a+b \cos \theta)\left(1-\cos \left(\phi_{i}-\phi\right)\right)\right\}^{3 / 2}} \mathrm{~d} \phi_{i}, \\
\left(h_{a}\right)_{3} & =\int_{0}^{2 \pi} \frac{a^{2}-a(a+b \cos \theta) \cos \left(\phi_{i}-\phi\right)}{\left\{b^{2}+2 a(a+b \cos \theta)\left(1-\cos \left(\phi_{i}-\phi\right)\right)\right\}^{3 / 2}} \mathrm{~d} \phi_{i} .
\end{aligned}
$$

Using the relations (65) in eqn. (32), variation in pressure work becomes

$$
\delta E_{p}=-\int_{0}^{2 \pi} \int_{0}^{2 \pi} \tilde{P}[\{(\tilde{a}+\tilde{b} \cos \theta) \tilde{b} \cos \theta\} \delta \tilde{a}+\{(\tilde{a}+\tilde{b} \cos \theta) \tilde{b}\} \delta \tilde{b}] \mathrm{d} \theta \mathrm{d} \phi .
$$

Multiplying and dividing the integrand by $\sqrt{G}$,

$$
\delta E_{p}=-\int_{0}^{2 \pi} \int_{0}^{2 \pi} \tilde{P} \sqrt{G}\left(\lambda_{1} \lambda_{2} \cos \theta \delta \tilde{a}+\lambda_{1} \lambda_{2} \delta \tilde{b}\right) \mathrm{d} \theta \mathrm{d} \phi .
$$

From the principle of minimum potential energy and eqns. (71), (74) and (78),

$$
\begin{aligned}
\int_{0}^{2 \pi} \int_{0}^{2 \pi}\left[\left(\frac{1}{R_{b}} \frac{\partial \widehat{U}}{\partial a}\right.\right. & \left.T \sqrt{G}-\frac{\chi}{2 R_{b}} \frac{\partial \mathcal{H}}{\partial a} T \sqrt{G}-\tilde{P} \sqrt{G} \lambda_{1} \lambda_{2} \cos \theta\right) \delta \tilde{a} \\
& \left.+\left(\frac{1}{R_{b}} \frac{\partial \widehat{U}}{\partial b} T \sqrt{G}-\frac{\chi}{2 R_{b}} \frac{\partial \mathcal{H}}{\partial b} T \sqrt{G}-\tilde{P} \sqrt{G} \lambda_{1} \lambda_{2}\right) \delta \tilde{b}\right] \mathrm{d} \theta \mathrm{d} \phi=0 .
\end{aligned}
$$

Since $\tilde{a}$ and $\tilde{b}$ are arbitrary, each of the coefficients of $\delta \tilde{a}$ and $\delta \tilde{b}$ is zero. Thus, we obtain the following governing equations

$$
\begin{array}{r}
\int_{0}^{2 \pi} \int_{0}^{2 \pi}\left(\frac{1}{R_{b}} \frac{\partial \widehat{U}}{\partial a} T \sqrt{G}-\frac{\chi}{2 R_{b}} \frac{\partial \mathcal{H}}{\partial a} T \sqrt{G}-\tilde{P} \sqrt{G} \lambda_{1} \lambda_{2} \cos \theta\right) \mathrm{d} \theta \mathrm{d} \phi=0 \\
\int_{0}^{2 \pi} \int_{0}^{2 \pi}\left(\frac{1}{R_{b}} \frac{\partial \widehat{U}}{\partial b} T \sqrt{G}-\frac{\chi}{2 R_{b}} \frac{\partial \mathcal{H}}{\partial b} T \sqrt{G}-\tilde{P} \sqrt{G} \lambda_{1} \lambda_{2}\right) \mathrm{d} \theta \mathrm{d} \phi=0
\end{array}
$$

Using eqns. (72), (73), and introducing $P=\tilde{P} R_{b} / C_{1} T$,

$$
\begin{aligned}
& \int_{0}^{2 \pi} \int_{0}^{2 \pi} \lambda_{2}\left(1+\alpha \lambda_{1}^{2}\right)\left(\frac{1}{1+r \cos \theta}\right)\left(1-\frac{1}{\lambda_{1}^{2} \lambda_{2}^{4}}\right) \sqrt{G} \mathrm{~d} \theta \mathrm{d} \phi \\
& +\int_{0}^{2 \pi} \int_{0}^{2 \pi}-\frac{\chi \mathcal{M}}{2} \frac{\partial \mathcal{H}}{\partial a} \sqrt{G} \mathrm{~d} \theta \mathrm{d} \phi+\int_{0}^{2 \pi} \int_{0}^{2 \pi}-\frac{P}{2} \lambda_{1} \lambda_{2} \cos \theta \sqrt{G} \mathrm{~d} \theta \mathrm{d} \phi=0 \\
& \int_{0}^{2 \pi} \int_{0}^{2 \pi} \lambda_{2}\left(1+\alpha \lambda_{1}^{2}\right)\left(\frac{\cos \theta}{1+r \cos \theta}\right)\left(1-\frac{1}{\lambda_{1}^{2} \lambda_{2}^{4}}\right) \sqrt{G} \mathrm{~d} \theta \mathrm{d} \phi \\
& +\int_{0}^{2 \pi} \int_{0}^{2 \pi} \frac{\lambda_{1}}{r}\left(1+\alpha \lambda_{2}^{2}\right)\left(1-\frac{1}{\lambda_{1}^{4} \lambda_{2}^{2}}\right) \sqrt{G} \mathrm{~d} \theta \mathrm{d} \phi \\
& +\int_{0}^{2 \pi} \int_{0}^{2 \pi}-\frac{\chi \mathcal{M}}{2} \frac{\partial \mathcal{H}}{\partial b} \sqrt{G} \mathrm{~d} \theta \mathrm{d} \phi+\int_{0}^{2 \pi} \int_{0}^{2 \pi}-\frac{P}{2} \lambda_{1} \lambda_{2} \sqrt{G} \mathrm{~d} \theta \mathrm{d} \phi=0
\end{aligned}
$$

where the partial derivatives of $\mathcal{H}$ are as defined in eqns. (75). The integrals are evaluated numerically using trapezoidal method (via trapz in MATLAB ${ }^{\circledR}$ ) with a sufficiently small spacing ensuring the convergence of the integral value. To solve the resulting algebraic equations in $a, b, P$, and $\mathcal{M}$ for a given value of $\varrho(0)=a+b$, we start by guessing the pair $(b, P)$. Nelder-Meads simplex search technique (via fminsearch in MATLAB ${ }^{\circledR}$ ) is used to obtain the correct pair that makes the left hand sides in the governing equations sufficiently small $\left(\mathcal{O}\left(10^{-13}\right)\right)$.

As mentioned earlier, centre of the parametrically deformed cross-section $(\tilde{a})$ is taken to be the position of the current carrying loop for the instant where the outer edges of the membrane according to both general and parametrically deformations coincide. Since the solution procedure starts with a value of $\varrho(0)$ as an input, it can be said that the outer edge of the deformed cross-section decides the placement of the wire. 


\section{Cauchy stresses}

Cauchy stresses for the magnetoelastic incompressible material under consideration, according to Barham et al. (2007) are as follows.

$$
\tilde{\boldsymbol{\sigma}}=\rho \psi_{\mathbf{F}} \mathbf{F}^{t}+\mu_{0}\left(\mathbf{h}_{a} \otimes \mathbf{h}_{a}-\frac{1}{2}\left|\mathbf{h}_{a}\right|^{2} \mathbf{I}\right)+\mu_{0} \mathbf{h}_{a} \otimes \mathbf{m}-q \mathbf{I} .
$$

where the operation $\otimes$ is defined as $(\boldsymbol{k} \otimes \boldsymbol{l}) \boldsymbol{m}=(\boldsymbol{l} \cdot \boldsymbol{m}) \boldsymbol{k}$, the free energy density $\rho \psi(\mathbf{F}, \boldsymbol{\mu})$ is as given in eqn. $(17)$ and $(\cdot)_{\mathbf{F}}=\partial(\cdot) / \partial \mathbf{F}$. We have

$$
\rho \psi_{\mathbf{F}} \mathbf{F}^{t}=\frac{\partial}{\partial \mathbf{F}}\left(\widehat{W}+\frac{1}{2} C|\boldsymbol{\mu}|^{2}\right) \mathbf{F}^{t}
$$

From the definitions (17) and (46),

$$
\rho \psi_{\mathbf{F}} \mathbf{F}^{t}=\widehat{W}_{\mathbf{F}} \mathbf{F}^{t}+\frac{\partial}{\partial \mathbf{F}}\left(\frac{1}{2} \chi C_{1} \mathcal{M}\left|\mathbf{h}_{a}\right|^{2}\right) \mathbf{F}^{t} .
$$

Noticing from eqn. (42) that the magnitude of the magnetic field intensity does not depend on the derivatives of the deformed coordinates and from eqns. (14),

$$
\begin{aligned}
\frac{\partial}{\partial \lambda_{1}}\left|\mathbf{h}_{a}\right|^{2} & =\left(\frac{\partial}{\partial \varrho^{\prime}} \frac{\partial \varrho^{\prime}}{\partial \lambda_{1}}+\frac{\partial}{\partial \eta^{\prime}} \frac{\partial \eta^{\prime}}{\partial \lambda_{1}}\right)\left|\mathbf{h}_{a}\right|^{2}=0, \\
\frac{\partial}{\partial \lambda_{2}}\left|\mathbf{h}_{a}\right|^{2} & =\left(\frac{\partial}{\partial \varrho} \frac{\partial \varrho}{\partial \lambda_{2}}\right)\left|\mathbf{h}_{a}\right|^{2}=(1+r \cos \theta) \frac{\partial}{\partial \varrho}\left|\mathbf{h}_{a}\right|^{2} .
\end{aligned}
$$

We can see from eqn. (15) that the only non-zero elements of the deformation gradient tensor are the principal stretches. Then,

$$
\begin{gathered}
\left(\rho \psi_{\mathbf{F}} \mathbf{F}^{t}\right)_{\theta}=\lambda_{1} \frac{\partial \widehat{W}}{\partial \lambda_{1}}+\lambda_{1} \frac{\partial}{\partial \lambda_{1}}\left(\frac{1}{2} \chi C_{1} \mathcal{M}\left|\mathbf{h}_{a}\right|^{2}\right)=\lambda_{1} \frac{\partial \widehat{W}}{\partial \lambda_{1}} \\
\left(\rho \psi_{\mathbf{F}} \mathbf{F}^{t}\right)_{\phi}=\lambda_{2} \frac{\partial \widehat{W}}{\partial \lambda_{2}}+\lambda_{2} \frac{\partial}{\partial \lambda_{2}}\left(\frac{1}{2} \chi C_{1} \mathcal{M}\left|\mathbf{h}_{a}\right|^{2}\right)=\lambda_{2} \frac{\partial \widehat{W}}{\partial \lambda_{2}}+\frac{1}{2} \varrho \chi C_{1} \mathcal{M} \frac{\partial}{\partial \varrho}\left|\mathbf{h}_{a}\right|^{2} .
\end{gathered}
$$

And,

$$
\begin{aligned}
\mathbf{h}_{a} \otimes \mathbf{m} & =\chi\left(\frac{I}{4 \pi R_{b}}\right)^{2}\left(\left(h_{a}\right)_{\theta} \widehat{\boldsymbol{g}}_{1}+\left(h_{a}\right)_{\xi} \widehat{\boldsymbol{g}}_{3}\right) \otimes\left(\left(h_{a}\right)_{\theta} \widehat{\boldsymbol{g}}_{1}+\left(h_{a}\right)_{\xi} \widehat{\boldsymbol{g}}_{3}\right), \\
\mathbf{h}_{a} \otimes \mathbf{h}_{a} & =\left(\frac{I}{4 \pi R_{b}}\right)^{2}\left(\left(h_{a}\right)_{\theta} \widehat{\boldsymbol{g}}_{1}+\left(h_{a}\right)_{\xi} \widehat{\boldsymbol{g}}_{3}\right) \otimes\left(\left(h_{a}\right)_{\theta} \widehat{\boldsymbol{g}}_{1}+\left(h_{a}\right)_{\xi} \widehat{\boldsymbol{g}}_{3}\right) .
\end{aligned}
$$

Thus, the above tensor products contribute, respectively, $\chi\left(h_{a}\right)_{\theta}^{2}$ and $\left(h_{a}\right)_{\theta}^{2}$ to the meridional stress, and $\chi\left(h_{a}\right)_{\xi}^{2}$ and $\left(h_{a}\right)_{\xi}^{2}$ to the thickness stress. The meridional, circumferential, and thickness stresses are given as

$$
\begin{aligned}
& \tilde{\sigma}_{\theta}=\left(\rho \psi_{\mathbf{F}} \mathbf{F}^{t}-q \mathbf{I}\right)_{\theta}+\mu_{0}\left(\left(\frac{I}{4 \pi R_{b}}\right)^{2}\left(h_{a}\right)_{\theta}^{2}-\frac{1}{2}\left|\mathbf{h}_{a}\right|^{2}\right)+\mu_{0} \chi\left(\frac{I}{4 \pi R_{b}}\right)^{2}\left(h_{a}\right)_{\theta}^{2}, \\
& \tilde{\sigma}_{\phi}=\left(\rho \psi_{\mathbf{F}} \mathbf{F}^{t}-q \mathbf{I}\right)_{\phi}-\frac{1}{2} \mu_{0}\left|\mathbf{h}_{a}\right|^{2}, \\
& \tilde{\sigma}_{\xi}=\left(\rho \psi_{\mathbf{F}} \mathbf{F}^{t}-q \mathbf{I}\right)_{\xi}+\mu_{0}\left(\left(\frac{I}{4 \pi R_{b}}\right)^{2}\left(h_{a}\right)_{\xi}^{2}-\frac{1}{2}\left|\mathbf{h}_{a}\right|^{2}\right)+\mu_{0} \chi\left(\frac{I}{4 \pi R_{b}}\right)^{2}\left(h_{a}\right)_{\xi}^{2} .
\end{aligned}
$$

and $q$, called the constraint pressure corresponding to the incompressibility condition $\lambda_{1} \lambda_{2} \lambda_{3}=1$ is obtained by setting the mechanical contribution to the thickness stress $\tilde{\sigma}_{\xi}$ to zero (Barham et al., 2007, eqn. (64)) i.e.,

$$
q=\lambda_{3} \frac{\partial \widehat{W}}{\partial \lambda_{3}}=\lambda_{3}\left(2 C_{1} \lambda_{3}-2 \frac{C_{2}}{\lambda_{3}^{3}}\right)=\frac{2 C_{1}}{\lambda_{1}^{2} \lambda_{2}^{2}}-2 C_{2} \lambda_{1}^{2} \lambda_{2}^{2} .
$$

We non-dimensionalise the above quantities by multiplying them with $\lambda_{3} / C_{1}$ (Pearce et al., 2011, Yang and Feng, 1970). The resulting non-dimensional meridional and circumferential Cauchy stresses are as follows.

$$
\begin{gathered}
\sigma_{\theta}=\frac{\lambda_{3}}{C_{1}}\left(\lambda_{1} \frac{\partial \widehat{W}}{\partial \lambda_{1}}-\lambda_{3} \frac{\partial \widehat{W}}{\partial \lambda_{3}}\right)+\frac{\lambda_{3}}{C_{1}} \mu_{0}(1+\chi)\left(\frac{I}{4 \pi R_{b}}\right)^{2}\left(h_{a}\right)_{\theta}^{2}+\frac{\lambda_{3}}{C_{1}}\left(-\frac{1}{2} \mu_{0}\left|\mathbf{h}_{a}\right|^{2}\right) \\
\sigma_{\phi}=\frac{\lambda_{3}}{C_{1}}\left(\lambda_{2} \frac{\partial \widehat{W}}{\partial \lambda_{2}}-\lambda_{3} \frac{\partial \widehat{W}}{\partial \lambda_{3}}\right)+\frac{\lambda_{3}}{C_{1}}\left(\frac{1}{2} \varrho \chi C_{1} \mathcal{M} \frac{\partial}{\partial \varrho}\left|\mathbf{h}_{a}\right|^{2}\right)+\frac{\lambda_{3}}{C_{1}}\left(-\frac{1}{2} \mu_{0}\left|\mathbf{h}_{a}\right|^{2}\right)
\end{gathered}
$$


Using the definitions (20) and (19), the non-dimensional stresses become

$$
\begin{aligned}
& \sigma_{\theta}=2\left(\frac{\lambda_{1}}{\lambda_{2}}-\frac{1}{\lambda_{1}^{3} \lambda_{2}^{3}}\right)\left(1+\alpha \lambda_{2}^{2}\right)+\frac{1}{\lambda_{1} \lambda_{2}} \mathcal{M}\left(\frac{1}{2}+\chi\right)\left(h_{a}\right)_{\theta}^{2}-\frac{1}{2 \lambda_{1} \lambda_{2}} \mathcal{M}\left(h_{a}\right)_{\xi}^{2}, \\
& \sigma_{\phi}=2\left(\frac{\lambda_{2}}{\lambda_{1}}-\frac{1}{\lambda_{1}^{3} \lambda_{2}^{3}}\right)\left(1+\alpha \lambda_{1}^{2}\right)+\frac{\varrho}{2 \lambda_{1} \lambda_{2}} \chi \mathcal{M} \frac{\partial}{\partial \varrho}\left(\left(h_{a}\right)_{\theta}^{2}+\left(h_{a}\right)_{\xi}^{2}\right)-\frac{1}{2 \lambda_{1} \lambda_{2}} \mathcal{M}\left(\left(h_{a}\right)_{\theta}^{2}+\left(h_{a}\right)_{\xi}^{2}\right) .
\end{aligned}
$$

where $\left(h_{a}\right)_{\xi}$ and $\left(h_{a}\right)_{\theta}$ are as defined in the expression (43).

\section{Results and discussion}

Table 1: Parameters used in the analyses unless mentioned otherwise.

\begin{tabular}{cc}
\hline$\alpha$ & $\chi$ \\
\hline 0.1 & 2.5 \\
\hline
\end{tabular}

The governing equations (55) and (56) for general deformation, and equations (82) and (83) for parametric deformation are solved numerically according to the respective solution procedures mentioned in Sections 2.4 and 3 respectively. We choose two different values for the non-dimensional radius of initial circular cross-section: $r=0.2$ and 0.5 to represent two distinct geometries of the toroidal membrane. Only non-dimensional quantities are used henceforth. We use the same values of the material parameter and magnetic susceptibility in our calculations that were used by Tamadapu and DasGupta (2014a) and Barham et al. (2008) and are listed in Table 1 .

\subsection{Validating the formulation}

To verify the authenticity of the formulation presented earlier, a comparison of our results with those obtained by Tamadapu and DasGupta (2014a) for free inflation of a purely elastic toroidal membrane is made. Figure 4 shows the comparison of the principal stretches $\left(\lambda_{1}, \lambda_{2}\right)$ and the non-dimensional Cauchy stresses $\left(\sigma_{\theta}, \sigma_{\phi}\right)$ where an appreciable matching of the numerical values can be seen. Regarding the derivation of the expression for magnetic field intensity (eqn. (38)), it was already noted that it is the same as that presented in (Grivich and Jackson, 2000). In addition, Figure 5 helps visualise the distribution of the magnetic field intensity $\left(\mathbf{h}_{a}\right)$. In this case, wire is placed at a radius $a=1.5$. The magnetic field lines are approximately circular around the wires. Field is stronger towards the inside of the torus $(\varrho \rightarrow 0)$ because of positive reinforcement while it becomes weaker on the outside as magnetic field lines from different parts of the current loop tend to cancel each other.

\subsection{Comparison of general and parametric deformations}

To verify the validity of the parametric deformation assumption, Table 2 presents the principal stretches $\left(\lambda_{1}\right.$ and $\left.\lambda_{2}\right)$ at the inner edge $(\theta=\pi)$ for various values of inflating gas pressure $(P)$ and initial radii of cross-section $(r)$. This particular location is chosen because of the maximum differences between the two cases. It is clear that the assumption does not work well for higher values of pressure and large initial radius of undeformed cross-section $(r)$.

We see that the absolute value of percentage error is greater in case of meridional stretch for a given pressure and radius of undeformed cross-section. Also, both the per cent errors increase in magnitude with increase in pressure for a given non-dimensional radius, and with increase in non-dimensional initial radius for a given inflating pressure. Since the non-dimensional quantity $r$ is the ratio of the smaller $\left(R_{s}\right)$ to bigger $\left(R_{b}\right)$ radii and the parametric deformation assumption is primarily concerned with the deformation of the membrane in meridional direction (keeping the shape circular), its effect is more pronounced in $\theta$ - direction and by extension in $\lambda_{1}$. The constraint becomes more burdensome for higher values of $r$ or $P$ when the cross-section has greater tendency to deviate from being circular. We can also see that the assumption underestimates meridional stretches while overestimating the circumferential ones. Since the deformation in $\theta-$ direction is restricted to circular cross-section, for a given pressure, the membrane tries to achieve equilibrium by increasing the bigger radius $\left(R_{b}\right)$ rather than the smaller one $\left(R_{s}\right)$. Note that the strain energy $E_{\lambda}$ can be increased by increasing either of the stretches: $\lambda_{1}$ and $\lambda_{2}$ (see relations (19)).

Figure 6 shows a few cross-sections during the inflation process, both for general and parametric deformations. Upper halves represent general and the lower halves parametric deformations. Since the solution procedure starts with a known value of the position of the outer edge $(\varrho(0))$ as an input, all the instances in Figure 6 are chosen such that the outer edges of the membrane coincide for easier comparison and also specify 


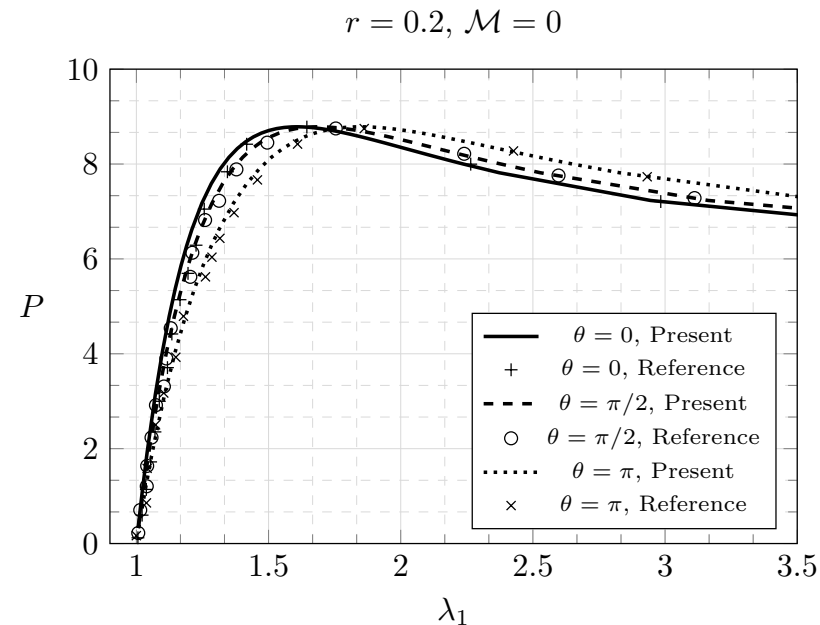

(a)

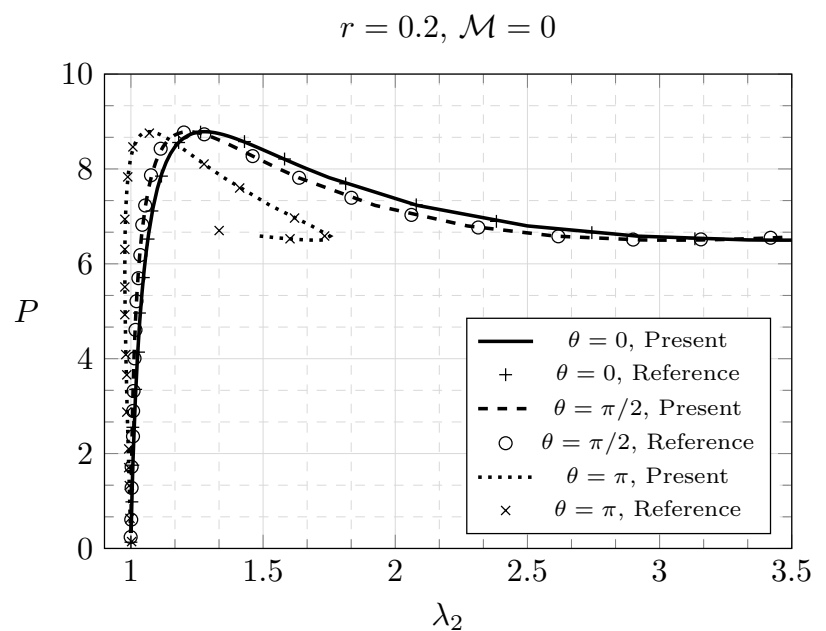

(b)

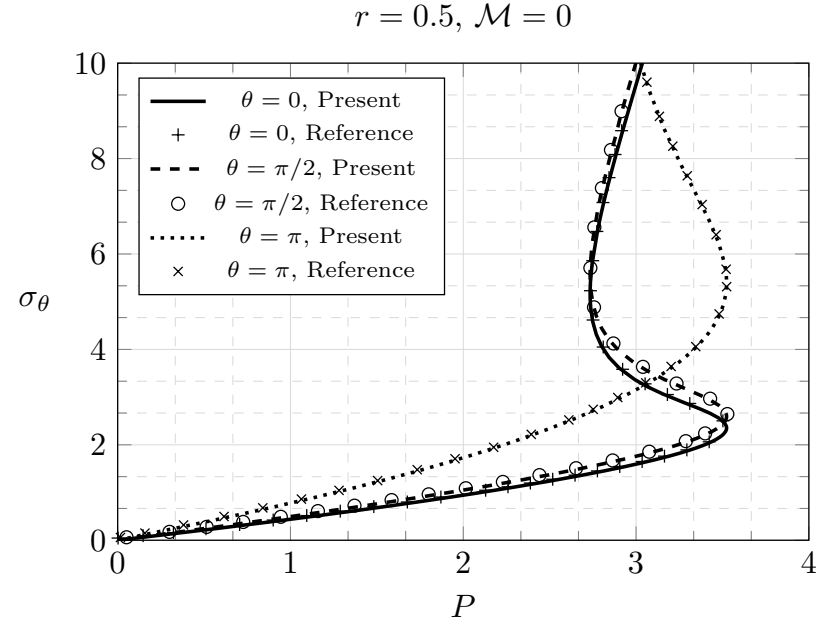

(c)

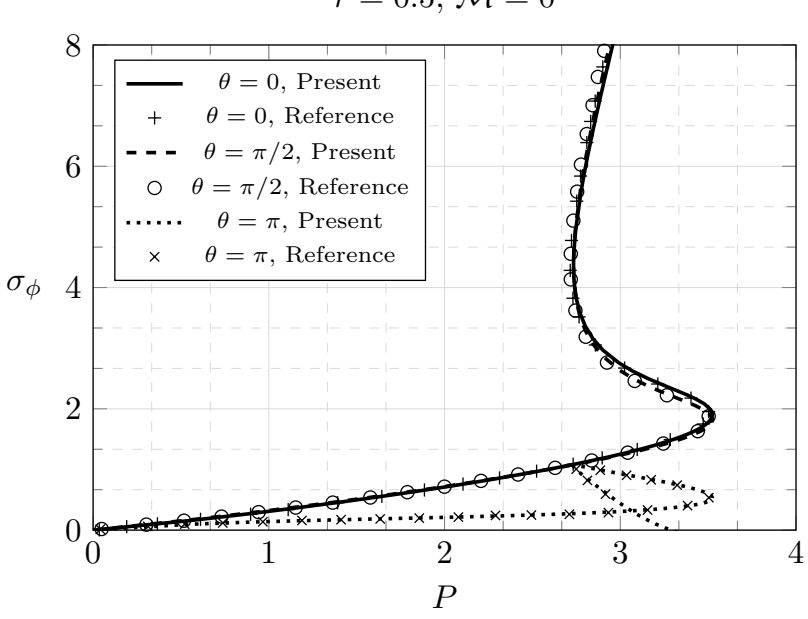

(d)

Figure 4: Comparison of the present results with those obtained by Tamadapu and DasGupta (2014a) for purely elastic case $(\mathcal{M}=0)$. 


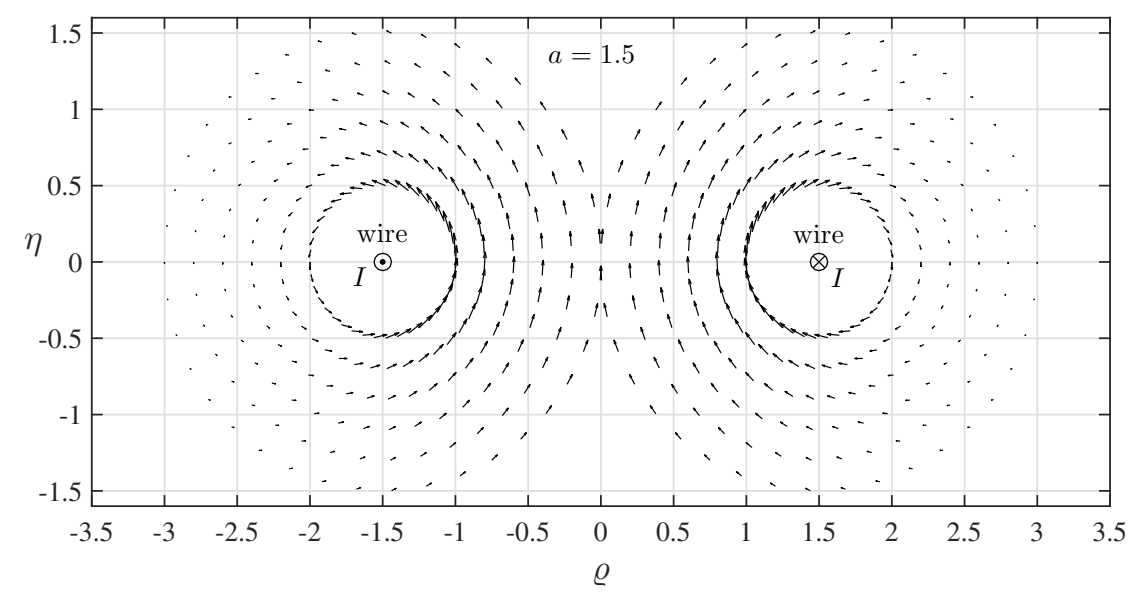

Figure 5: Distribution of the magnetic field intensity for the arrangement chosen. This confirms with the physics of the situation.

the position of the current carrying loop - centre of the circular cross-section. However, the inflating pressure corresponding to these two profiles need not be the same. It can be observed that the cross-section in general deformation remains fairly circular in the beginning while deviating from the assumption as pressure increases. In the presence of an external magnetic field, this deviation becomes significant even at lower pressures. Also observe that the membrane with different radii of initial cross-section behave differently upon inflation. In Figure 6, upon inflation, the inner edge moves inward (towards the origin; see Figure 1) for $r=0.5$ while for $r=0.2$, it moves outward until certain pressure and then starts moving inward. We attribute this behaviour to the geometrical properties inherent to a toroid. See (Tamadapu and DasGupta, 2014a) for a brief explanation of such a geometrical connection.

Table 2: Comparison of the circular cross-section assumption with the general deformation for purely elastic case $(\mathcal{M}=0)$. The assumption does not seem to hold well for higher pressures or radii of cross-section.

\begin{tabular}{cc|ccc|ccc}
\hline$P$ & $r$ & $\begin{array}{c}\lambda_{1} \\
\text { parametric }\end{array}$ & $\begin{array}{c}\lambda_{1}(\theta=\pi) \\
\text { general }\end{array}$ & $\begin{array}{c}\text { error in } \\
\lambda_{1}(\%)\end{array}$ & $\begin{array}{c}\lambda_{2}(\theta=\pi) \\
\text { parametric }\end{array}$ & $\begin{array}{c}\lambda_{2}(\theta=\pi) \\
\text { general }\end{array}$ & $\begin{array}{c}\text { error in } \\
\lambda_{2}(\%)\end{array}$ \\
\hline & 0.2 & 1.0489 & 1.0627 & -1.3000 & 0.9918 & 0.9903 & 0.1454 \\
2.0 & 0.3 & 1.0749 & 1.1128 & -3.4127 & 0.9813 & 0.9755 & 0.5946 \\
& 0.4 & 1.1015 & 1.1842 & -6.9803 & 0.9662 & 0.9504 & 1.6526 \\
& 0.5 & 1.1293 & 1.2893 & -12.4084 & 0.9451 & 0.9113 & 3.7058 \\
\hline & 0.2 & 1.0631 & 1.0811 & -1.6648 & 0.9899 & 0.9878 & 0.2113 \\
2.5 & 0.3 & 1.0989 & 1.1496 & -4.4102 & 0.9768 & 0.9685 & 0.8594 \\
& 0.4 & 1.1385 & 1.2521 & -9.0752 & 0.9572 & 0.9348 & 2.3971 \\
& 0.5 & 1.1851 & 1.4133 & -16.1481 & 0.9282 & 0.8805 & 5.4236 \\
\hline & 0.2 & 1.0783 & 1.1007 & -2.0426 & 0.9882 & 0.9855 & 0.2797 \\
3.0 & 0.3 & 1.1261 & 1.1911 & -5.4600 & 0.9726 & 0.9617 & 1.1400 \\
& 0.4 & 1.1846 & 1.3357 & -11.3124 & 0.9483 & 0.9188 & 3.2059 \\
& 0.5 & 1.2685 & 1.5915 & -20.2947 & 0.9088 & 0.8464 & 7.3788 \\
\hline
\end{tabular}

\subsection{Limit points}

We now study the typical behaviour of an inflating magnetoelastic toroidal membrane in the presence of a non-uniform magnetic field. Figure 7 shows one such variation of the principal stretches $\left(\lambda_{1}\right.$ and $\left.\lambda_{2}\right)$ for an initial radius of circular cross-section $r=0.5$ and non-dimensional magnetic energy parameter $\mathcal{M}=2 \times 10^{-4}$. Position of the current carrying wire, $\tilde{a}$ in a general deformed state (characterised by the distance of the outer edge of the membrane from the origin, $\varrho(0))$ is obtained using cubic interpolation of the data obtained for the parametric case where the wire is always at the centre of the deformed cross-section (see section 3). As is the case for any inflating nonlinear elastic membrane, principal stretches increase slowly with pressure until the critical or limit point after which the membrane flows much more freely even at lower pressures. We observe that no stable equilibrium (sometimes even unstable equilibrium) exists until a certain minimum pressure is 


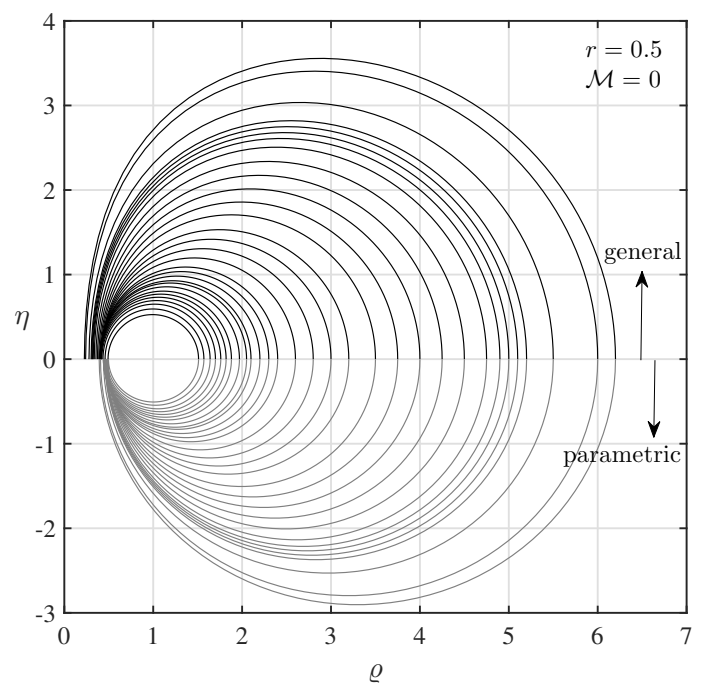

(a)

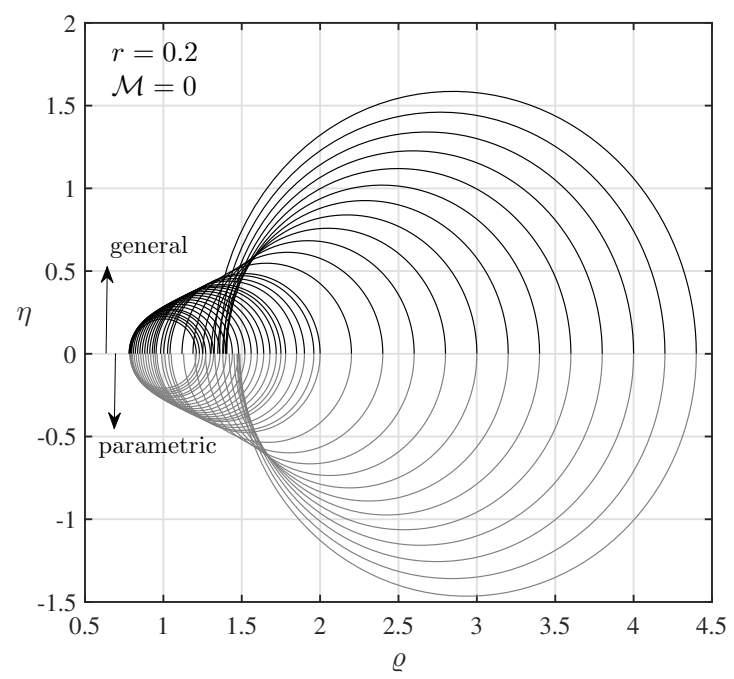

(b)

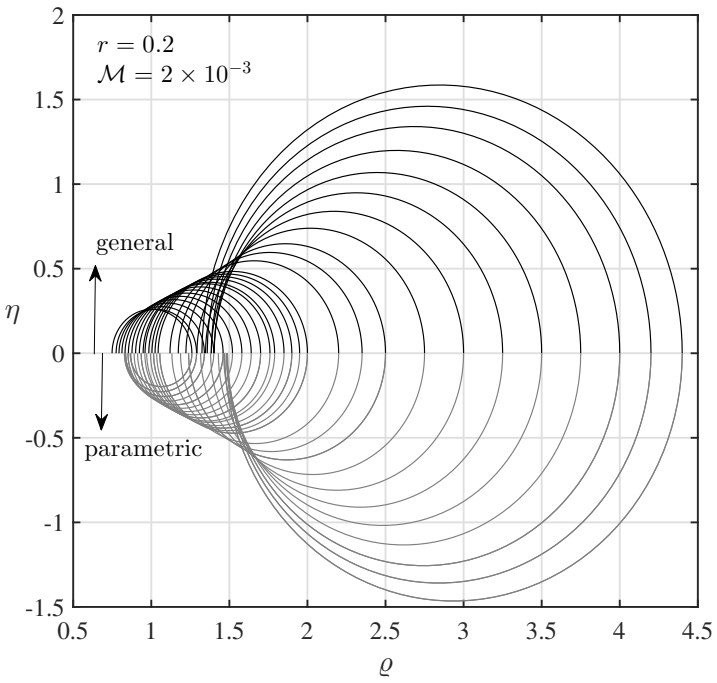

(c)

Figure 6: Inflation process for (a) $r=0.5$, purely elastic, (b) $r=0.2$, purely elastic, and (c) $r=0.2, \mathcal{M}=$ $2 \times 10^{-3}$. Upper half represents general deformation and the lower half parametric deformation. For a given position of the outer edge, wire is at the centre of the parametrically deformed cross-section (lower half). Various curves represent the symmetric half profiles of the deformed cross-sections for different values of the inflating pressure. 


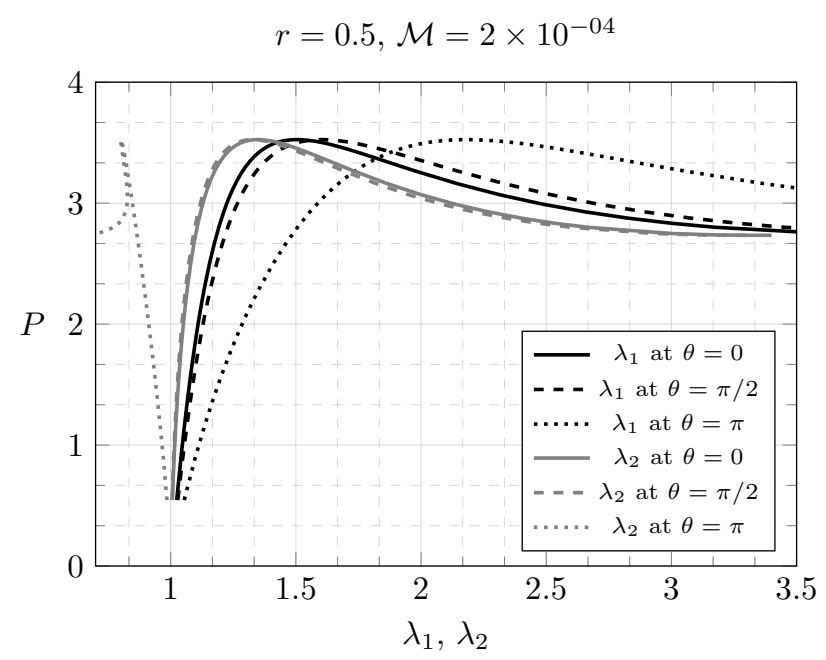

Figure 7: Variation of principal stretches upon inflation in the presence of a magnetic field $\left(\mathcal{M}=2 \times 10^{-04}\right)$. Initial radius of cross-section $r=0.5$. Position of wire is determined by the outer edge of the deformed crosssection $(\varrho(\theta=0))$ as mentioned before.

applied in the membrane. In Figure 7, inflation process starts from a non-zero value of the internal pressure (around 0.6). This point can be termed as a 'magnetic limit point'. Also, the effect of the magnetic field can be barely felt at higher values of the stretches since the magnetic field density dies down with the distance between the wire and a point in the membrane.

Figure 8 shows the effect of the external magnetic field on the principal stretches. Notice that with an increase in the magnetic field density at lower pressures, circumferential stretch at the inner edge $\lambda_{2}(\theta=\pi)$ increases while the others decrease (see the curves corresponding to $\mathcal{M}=0$ and $5 \times 10^{-4}$ for $r=0.2, \mathcal{M}=0$ and $5 \times 10^{-3}$ for $r=0.5$ ). Also, limit-point pressure increases with $\mathcal{M}$ because the applied magnetic field shrinks the magnetoelastic membrane while pressure relieves it. For visualisation, imagine a toroid with the radius of the centre-line, $a$ fixed while the radius of cross-section, $b$ is free to change. Application of magnetic field at no pressure shrinks the cross-section making the inner equator (at $\theta=\pi$ ) move outward and the outer equator move inward (at $\theta=0$ ) thus making the circumferential stresses at the outer edge compressive and those at the inner edge tensile in nature. Now, this trend can be reversed by inflating the membrane with an internal pressure $P$ as depicted in Figure $9 \mathrm{a}$ and Figure 9c. Only the circumferential stresses are shown since the changes in meridional stresses are found to be not significant compared to those in circumferential stresses. We attribute this to the geometry of the toroid: numerical value of the non-dimensional quantity $r$ is less than unity i.e., span of the toroid in $\phi$ - direction is much larger compared to that in $\theta$ - direction. For bigger values of $r$, the disparity seems to be mitigated. Also, the presence of magnetic field has maximum effect on the inner edge because the magnetic field is stronger at this location (see Figure 5).

Since a membrane cannot sustain compressive stresses, according to tension field theory (Steigmann, 1990, 2004), it can be said that a sufficiently strong external magnetic field can cause wrinkling on the outer edge of a toroidal membrane at lower pressures. We note the departure from the purely elastic case in which wrinkling usually tends to occur at the inner edge (Tamadapu and DasGupta, 2014a).

Another interesting phenomenon depicted in Figure 8 is the presence of more than one or a complete absence of limit point pressures. For sufficiently strong magnetic fields, the curves in Figure 8a see a drop in pressure twice. As mentioned earlier, external magnetic field tends to shrink the cross-section while the pressure tends to inflate it. At smaller radii of cross-sections the magnetic loading dominates the pressure loading. Although the cause of first limit point is not immediately apparent, it can be attributed to the loss of dominance of magnetic energy to the elastic energy and pressure. The second limit point is similar to the classical behaviour of membranes under high pressure.

We also observe from Figure $8 \mathrm{~b}$ that in the case of large $\mathcal{M}$, the required value of equilibrium pressure is already beyond the limit point pressure (for example, see the curves corresponding to $\theta=0, \mathcal{M}=0.04$ ). Peculiar behaviour of the toroidal membrane at the inner edge $(\theta=\pi)$ such as the one shown in Figure $8 \mathrm{~b}$ for $r=0.5$ as compared to that at other locations or radii of cross-section has a geometrical connection explained in Tamadapu and DasGupta (2014a). Briefly, the Gaussian curvature is positive in the range $\theta \in(-\pi / 2, \pi / 2)$ and negative otherwise.

Other observations from Figure 8 include the non-existence of multiple limit points and requirement of higher currents for larger radii of cross-section (in this case $r=0.5$ ). This can again be attributed to the 

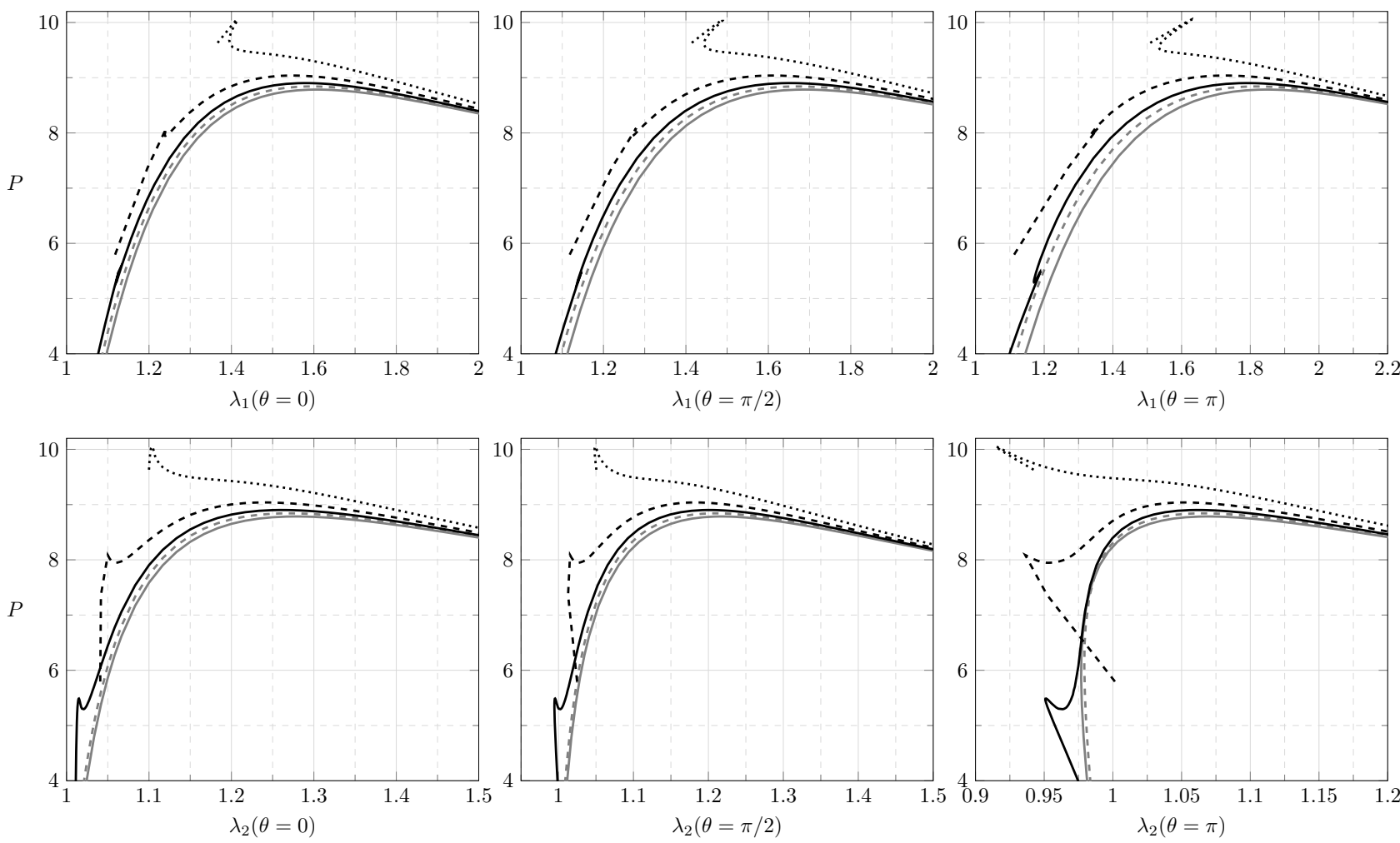

$\mathcal{M}=0-\cdots \mathcal{M}=5 \times 10^{-04}-\mathcal{M}=1 \times 10^{-03}---\mathcal{M}=2 \times 10^{-03} \ldots \ldots \quad \mathcal{M}=4 \times 10^{-03}$

(a)
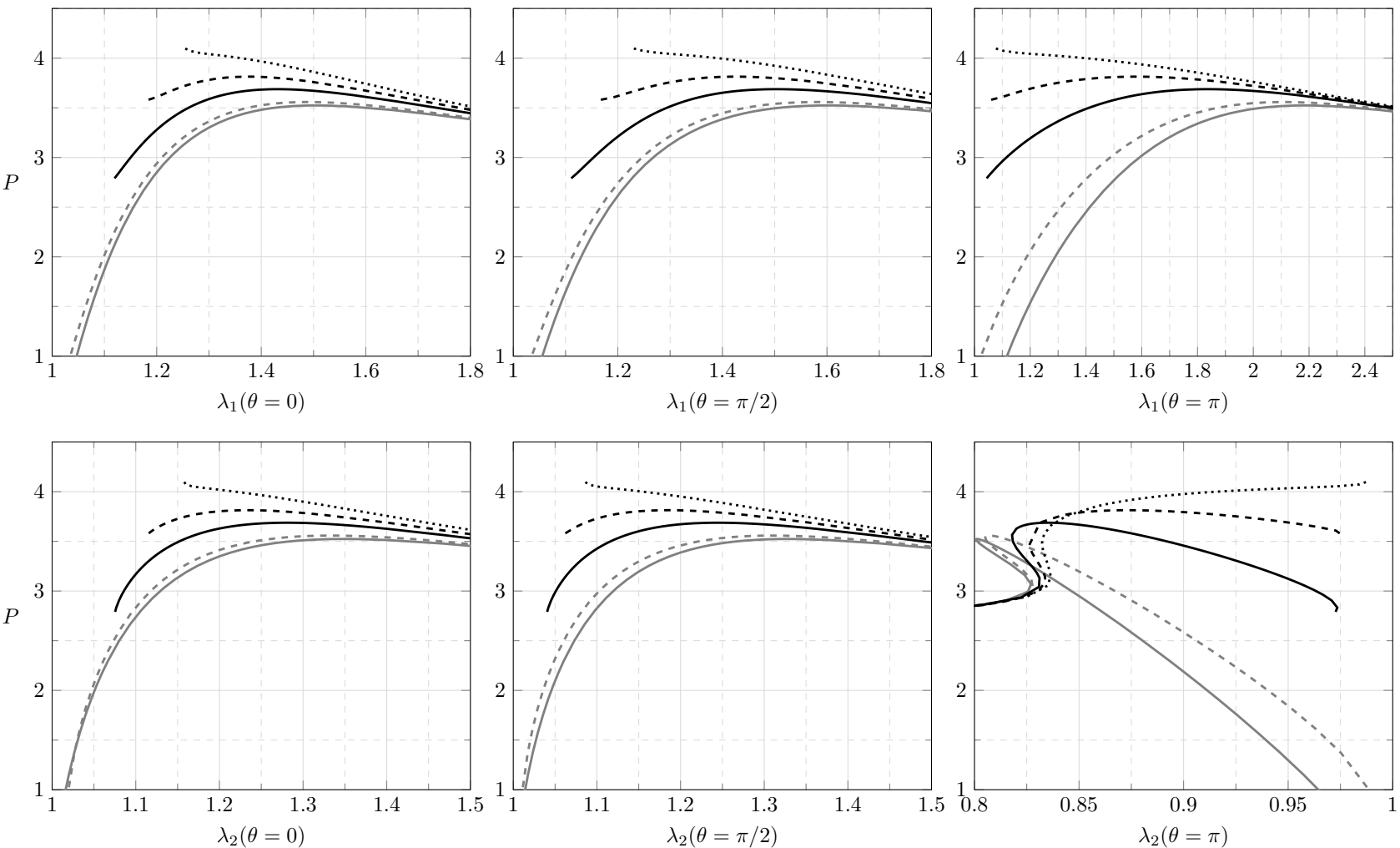

$-\mathcal{M}=0 \cdots \mathcal{M}=5 \times 10^{-03}-\mathcal{M}=2 \times 10^{-02} \quad \cdots \quad \mathcal{M}=3 \times 10^{-02} \quad \cdots \cdots \quad \mathcal{M}=4 \times 10^{-02}$

(b)

Figure 8: Variation of non-dimensional inflation pressure with principal stretches for initial radii of cross-section (a) $r=0.2$ and (b) $r=0.5$. Note the behaviour of critical points with magnetic energy parameter $\mathcal{M}$ and radius of cross-section $r$ 


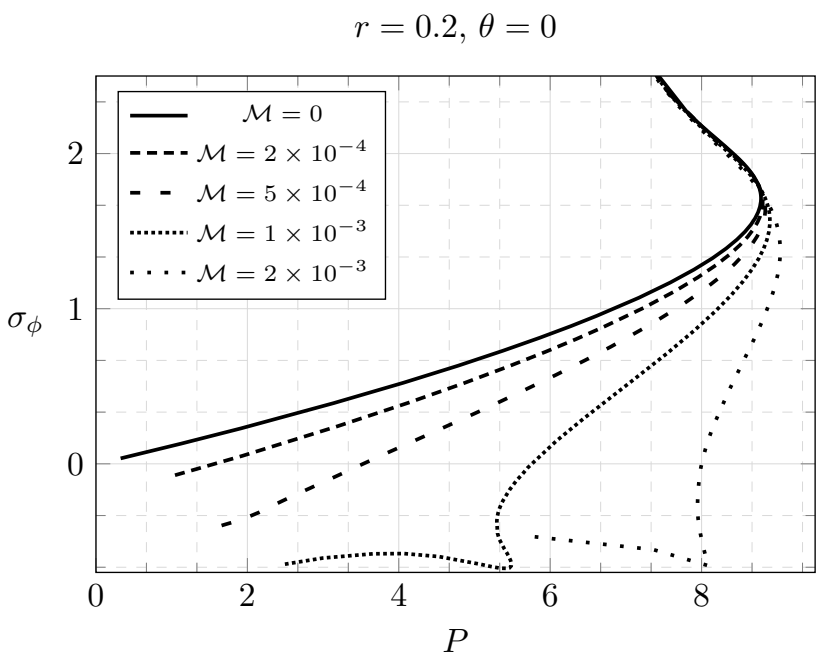

(a)

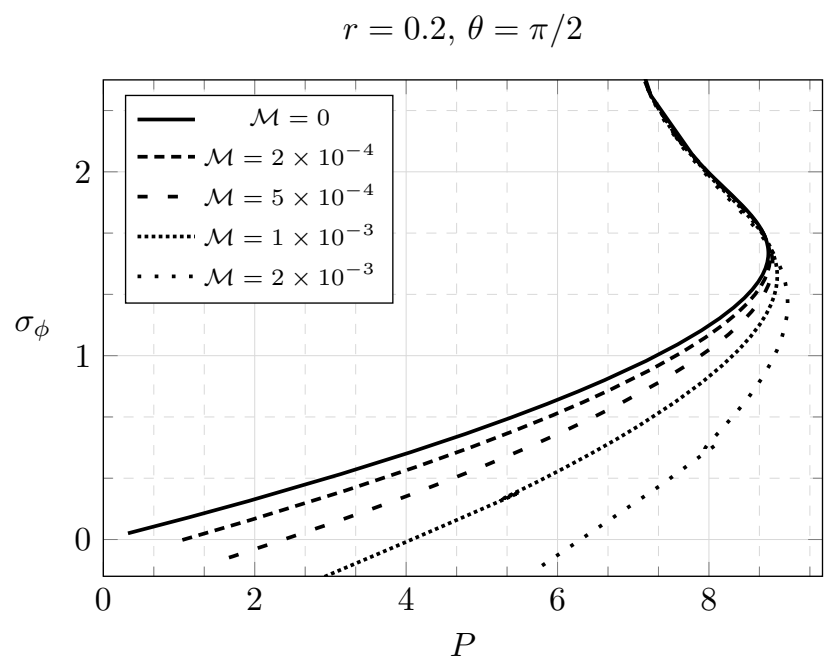

(b)

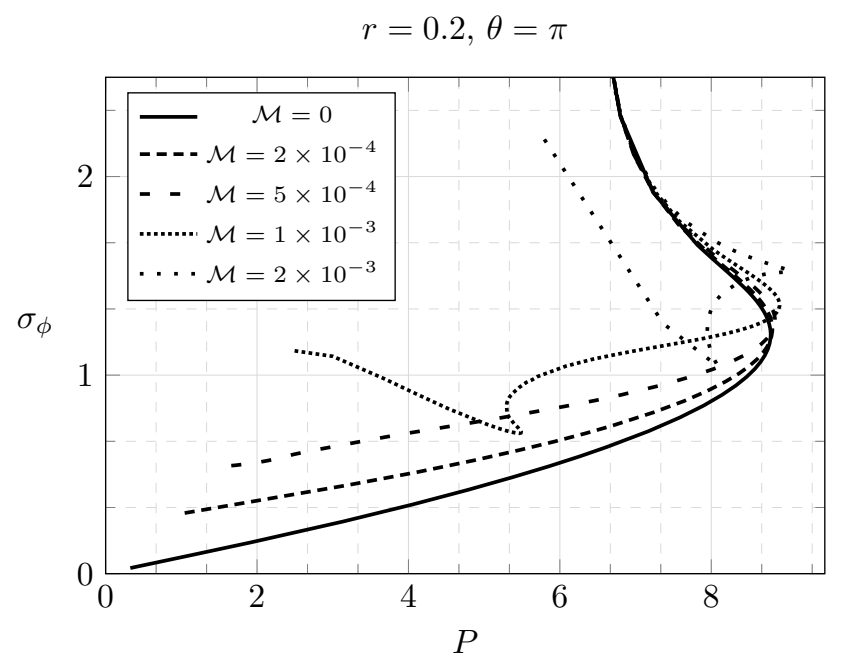

(c)

Figure 9: Variation of non-dimensional circumferential stresses with non-dimensional inflation pressure for initial radii of cross-section $r=0.2$. 
geometry of a toroid. Also observe from Figure 8 a that the curves for $\mathcal{M}=1 \times 10^{-3}$ and $\mathcal{M}=2 \times 10^{-3}$ cut the others corresponding to lower currents (or $\mathcal{M})$. This is due to the internal adjustment between the current carrying wire and the membrane at lower pressure in their attempt to find a stable equilibrium state close to the magnetic limit point and is of no physical value. In terms of the numerical methods used, as mentioned earlier, position of the current carrying wire $(a)$ is obtained by cubic extrapolation of the corresponding data obtained for parametric deformation case. This extrapolation may be the reason for this behaviour.

\subsection{Multiple equilibrium states}
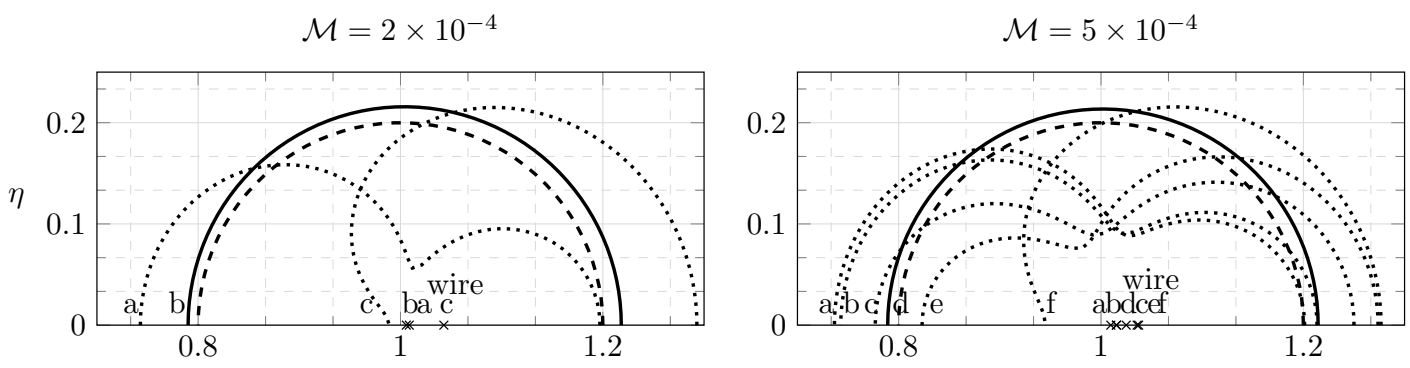

$$
\mathcal{M}=1 \times 10^{-3}
$$
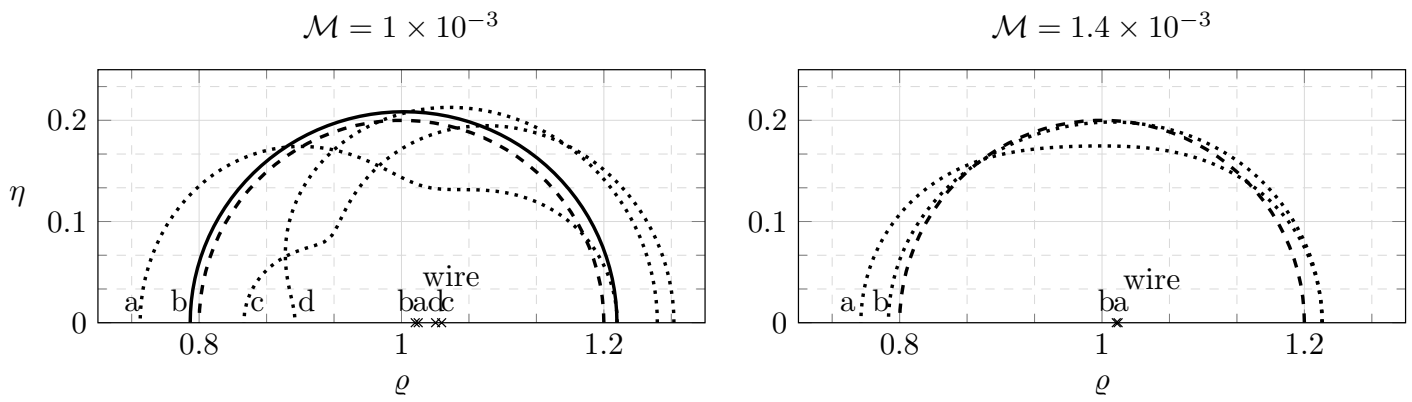

Figure 10: Possible deformed configurations of a toroidal membrane with initial radius of cross-section $r=0.2$, non-dimensional gas pressure $P=3.0$, and magnetic loading parameter $\mathcal{M}=2 \times 10^{-4}, 5 \times 10^{-4}, 1 \times 10^{-3}$ and $1.4 \times 10^{-3}$. Note that no equilibrium-stable or unstable-exists for larger values of $\mathcal{M}$. Each configuration has its own position for the current carrying wire. Solid and dotted lines stand for stable and unstable equilibria respectively and the dashed line for the reference configuration. Lower case alphabets associate the positions of the current carrying wire (marked as $\times$ ) with the respective deformed configurations.

We observed that multiple solutions of the governing equations exist for a given parameter $-P$ or $\varrho(0)$. Since the total energy functional depends on pressure, magnetic field, and displacements, multiple stationary points are bound to occur. Figure 10 shows a few such admissible solutions for initial radius $r=0.2$ and various values of the magnetic energy parameter. Stability of a solution is evaluated using the criterion described in Section 2.5. While the purely elastic case $(\mathcal{M}=0)$ has only one solution (a stable one, to be precise; not shown here), the number of equilibrium states increases until a certain value of $\mathcal{M}$ and then decreases until there are none. As can be observed from Figure 10, we do not obtain any stable solutions for the highest value of $\mathcal{M}=1.4 \times 10^{-3}$ and no solutions at all for $\mathcal{M}$ sufficiently greater than that value. As introduced in Section 5.3 we refer to this point of vanishing of equilibria as the magnetic limit point which was also seen in Figure 7 and Figure 8 . While in this particular example, not more than one stable equilibria are obtained from our numerical calculations, we cannot comment on the uniqueness of stable equilibrium state without a proper mathematical analysis that is beyond the scope of this paper.

\subsection{Stationary current carrying loop}

In all the previous analyses, the current carrying loop is placed based on the position of the outer edge, $\varrho(0)$. In this section, we study the inflation process keeping the wire stationary, for instance, at a radius $a=1.03$. Figure 11 shows some cross-sections at the end of the inflation process i.e., maximum possible internal pressures. Upon further increase in $P$, the membrane starts to bend in the middle towards the stationary wire. The bending deepens with increase in pressure until only unstable equilibrium states are possible and a further increase in pressure leads to a magnetic limit point where no equilibrium state, either stable or unstable, exists. We would like to mention that the stability criterion mentioned in Section 2.5 is incapable of determining the stability of some of the deformed configurations (dashed lines) in Figure 11. These configurations are found to satisfy just the necessary condition (see Section 2.5). More elaborate analysis is required to study their stability and is beyond the scope of the present study. 


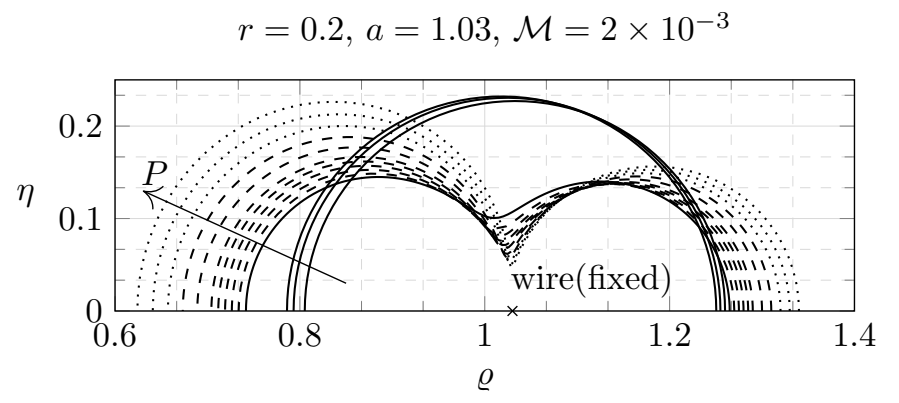

Figure 11: Membrane profiles for possible deformed configurations for $r=0.2, \mathcal{M}=2 \times 10^{-3}$ with the current carrying wire arbitrarily fixed at $a=1.03$. Each instant has its own gas pressure value. The arrow indicates the direction of increase of inflation pressure. Solid and dotted curves stand for stable and unstable equilibrium states, respectively. Stability is undetermined for the dashed curves for intermediate values of pressure.

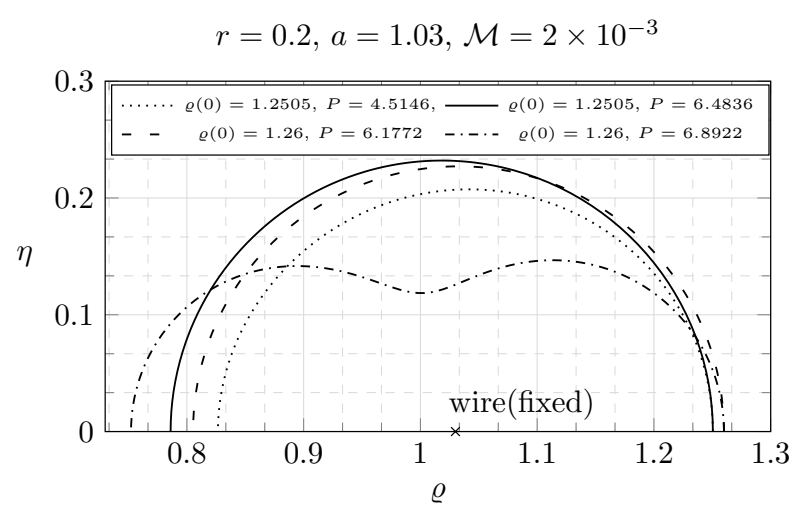

Figure 12: Membrane profiles for two equilibrium states each for the position of outer edge: $\varrho(0)=1.2505,1.26$ under the conditions: $r=0.2, \mathcal{M}=2 \times 10^{-3}$ with the current carrying wire arbitrarily fixed at $a=1.03$. Stability of the deformed state shown as a dotted line (for $\varrho(0)=1.2505, P=4.5146$ ) is undetermined. The other three curves correspond to stable states. 
A comment on the numerical computation scheme is in order. It is observed that for a given starting value of $\varrho(0)$, multiple equilibrium states are possible that correspond to different values of pressure. Four such equilibrium states are shown in Figure 12, two each for the two values of the position of the outer edge of the deformed configuration: $\varrho(0)=1.2505$ and $\varrho(0)=1.26$. Note that, unlike our previous observations, both the equilibrium states corresponding to $\varrho(0)=1.26$ are stable while the stability of one of the deformed states for $\varrho(0)=1.2505$ (dashed curve) remains currently undetermined.

\section{Conclusions}

Energy formulation based on magnetization is used to study the inflation of an incompressible nonlinear magnetoelastic toroidal membrane loaded by an internal pressure and a non-uniform magnetic field generated by a circular current carrying loop. It was found that a sufficiently large magnetic field can lead to more than one or a complete absence of limit points based on the dimensions of the undeformed membrane and can also cause wrinkling at the outer edge for lower inflating pressures. The presence of magnetic field can lead to multiple solutions of the governing equations for a given loading condition. Stability of each of these configurations is determined using the criterion described in Section 2.5. A further increase in the magnetic field can lead to a state where no equilibrium, either stable or unstable, exists which we referred to as a magnetic limit point. The computational procedure can sometimes lead to multiple stable equilibrium solutions for a given $\varrho(0)$ as shown in Section 5.5.

For the simplicity of analysis, it is assumed in this paper that the nonlinear deformation of the membrane has no influence on the surrounding magnetic field. Relaxation of this assumption to account for a general nonlinear magnetoelastic coupling will be carried out in a future work.

\section{Acknowledgements}

This work was financially supported by a Ramanujan fellowship and research grant by the Science and Engineering Research Board (SERB).

The authors would like to thank B. L. Sharma (IIT Kanpur) and G. Tamadapu (IIT Madras) for helpful discussions.

\section{References}

Alexander, Harold (1971), "Tensile instability of initially spherical balloons." International Journal of Engineering Science, 9, 151-160.

Barham, M., D. J. Steigmann, M. McElfresh, and R. E. Rudd (2007), "Finite deformation of a pressurized magnetoelastic membrane in a stationary dipole field." Acta Mechanica, 191, 1-19.

Barham, M., D. J. Steigmann, M. McElfresh, and R. E. Rudd (2008), "Limit-point instability of a magnetoelastic membrane in a stationary magnetic field." Smart Materials and Structures, 17, 055003.

Benedict, Robert, Alan Wineman, and Wei H Yang (1979), "The determination of limiting pressure in simultaneous elongation and inflation of nonlinear elastic tubes." International Journal of Solids and Structures, 15, 241-249.

Bonadies, Manuelita (1987), "Asymptotic behaviour of inflated toroidal membranes." Rendiconti del Seminario Matematico Università e Politecnico di Torino, 45 (3), 63-74.

Bonet, J., R. D. Wood, J. Mahaney, and P. Heywood (2000), "Finite element analysis of air supported membrane structures." Computer Methods in Applied Mechanics and Engineering, 190, 579-595.

Böse, H and R Röder (2009), "Magnetorheological elastomers with high variability of their mechanical properties." Journal of Physics: Conference Series, 149, 012090.

Böse, Holger, Raman Rabindranath, and Johannes Ehrlich (2012), "Soft magnetorheological elastomers as new actuators for valves." Journal of Intelligent Material Systems and Structures, 23, 989-994.

Bossis, G, C Abbo, S Cutillas, S Lacis, and C Métayer (2001), "Electroactive and electrostructured elastomers." International Journal of Modern Physics B, 15, 564-573.

Brigadnov, I A and A Dorfmann (2003), "Mathematical modeling of magneto-sensitive elastomers." International Journal of Solids and Structures, 40, 4659-4674.

Brown, William Fuller (1966), Magnetoelastic Interactions, volume 9. Springer, New York. 
Bustamante, R. (2010), "Transversely isotropic nonlinear magneto-active elastomers." Acta Mechanica, 210, $183-214$.

Castañeda, P Ponte and E Galipeau (2011), "Homogenization-based constitutive models for magnetorheological elastomers at finite strain." Journal of the Mechanics and Physics of Solids, 59, 194-215.

Castañeda, P Ponte and MH Siboni (2012), "A finite-strain constitutive theory for electro-active polymer composites via homogenization." International Journal of Non-Linear Mechanics, 47, 293-306.

Charrier, JM, S Shrivastava, and R Wu (1989), "Free and constrained inflation of elastic membranes in relation to thermoforming - non-axisymmetric problems." The Journal of Strain Analysis for Engineering Design, $24,55-74$.

Chatzigeorgiou, G., A. Javili, and P. Steinmann (2014), "Unified magnetomechanical homogenization framework with application to magnetorheological elastomers." Mathematics and Mechanics of Solids, 19, 193-211.

Danas, K., S. V. Kankanala, and N. Triantafyllidis (2012a), "Experiments and modeling of iron-particle-filled magnetorheological elastomers." Journal of the Mechanics and Physics of Solids, 60, 120-138.

Danas, Kostas, SV Kankanala, and Nicolas Triantafyllidis (2012b), "Experiments and modeling of iron-particlefilled magnetorheological elastomers." Journal of the Mechanics and Physics of Solids, 60, 120-138.

Dorfmann, A. and R. W. Ogden (2003), "Magnetoelastic modelling of elastomers." European Journal of Mechanics- A/Solids, 22, 497-507.

Dorfmann, A and RW Ogden (2005), "Some problems in nonlinear magnetoelasticity." Zeitschrift für angewandte Mathematik und Physik, 56, 718-745.

Dorfmann, L. and R. W. Ogden (2014), Nonlinear Theory of Electroelastic and Magnetoelastic Interactions. Springer, New York.

Ethiraj, G. and C. Miehe (2016), "Multiplicative magneto-elasticity of magnetosensitive polymers incorporating micromechanically-based network kernels." International Journal of Engineering Science, 102, 93 - 119.

Farshad, M and Andre Benine (2004), "Magnetoactive elastomer composites." Polymer Testing, 23, 347-353.

Feng, WW and W-H Yang (1973), "On the contact problem of an inflated spherical nonlinear membrane." Journal of Applied Mechanics, 40, 209-214.

Fox, JW and NC Goulbourne (2008), "On the dynamic electromechanical loading of dielectric elastomer membranes." Journal of the Mechanics and Physics of Solids, 56, 2669-2686.

Fox, JW and NC Goulbourne (2009), "Electric field-induced surface transformations and experimental dynamic characteristics of dielectric elastomer membranes." Journal of the Mechanics and Physics of Solids, 57, 14171435 .

Fried, Isaac (1982), "Finite element computation of large rubber membrane deformations." International Journal for Numerical Methods in Engineering, 18, 653-660.

Fulton, J. P. and J. Simmonds (1986), "Large deformations under vertical edge loads of annular membranes with various strain energy densities." International Journal of Non-Linear Mechanics, 21, 257-267.

Galipeau, E. and P. P. Castañeda (2013), "Giant field-induced strains in magnetoactive elastomer composites." Proceedings of the Royal Society A, 469, 20130385.

Gelfand, IM and SV Fomin (2000), Calculus of Variations,(Translated and edited by Silverman, RA). Dover Edition.

Ginder, JM, SM Clark, WF Schlotter, and ME Nichols (2002), "Magnetostrictive phenomena in magnetorheological elastomers." International Journal of Modern Physics B, 16, 2412-2418.

Ginder, John M, Mark E Nichols, Larry D Elie, and Janice L Tardiff (1999), "Magnetorheological elastomers: properties and applications." In 1999 Symposium on Smart Structures and Materials, 131-138, International Society for Optics and Photonics.

Ginder, John M, William F Schlotter, and Mark E Nichols (2001), "Magnetorheological elastomers in tunable vibration absorbers." In SPIE's 8th Annual International Symposium on Smart Structures and Materials, 103-110, International Society for Optics and Photonics. 
Gong, XL, XZ Zhang, and PQ Zhang (2005), "Fabrication and characterization of isotropic magnetorheological elastomers." Polymer Testing, 24, 669-676.

Grivich, Matthew I and David P Jackson (2000), "The magnetic field of current-carrying polygons: An application of vector field rotations." American Journal of Physics, 68, 469-474.

Grossman, Gershon (1991a), "Analysis of rim supports for off-axis inflatable reflectors. i: Loads." Journal of Aerospace Engineering, 4, 47-66.

Grossman, Gershon (1991b), "Analysis of rim supports for off-axis inflatable reflectors. ii: Deformations." Journal of Aerospace Engineering, 4, 67-77.

Grossman, Gershon (1994), "Tension element to reduce loads in rim support of inflatable reflector." Journal of Aerospace Engineering, 7, 129-142.

Haldar, K, B Kiefer, and A Menzel (2016), "Finite element simulation of rate-dependent magneto-active polymer response." Smart Materials and Structures, 25, 104003.

Hill, J. M. (1980), "The finite inflation of a thick-walled elastic torus." The Quarterly Journal of Mechanics and Applied Mathematics, 33, 471-490.

Holmes, Hal R, Ee Lim Tan, Keat Ghee Ong, and Rupak M Rajachar (2012), "Fabrication of biocompatible, vibrational magnetoelastic materials for controlling cellular adhesion." Biosensors, 2, 57-69.

Humphrey, J.D. (2003), "Review paper: Continuum biomechanics of soft biological tissues." Proceedings of the Royal Society of London A: Mathematical, Physical and Engineering Sciences, 459, 3-46.

Itskov, Mikhail and Vu Ngoc Khim (2016), "A polyconvex anisotropic free energy function for electro- and magneto-rheological elastomers." Mathematics and Mechanics of Solids, 21, 1126-1137.

Jenkins, Chris H and John W Leonard (1991), "Nonlinear dynamic response of membranes: state of the art." Applied Mechanics Reviews, 44, 319-328.

Jiang, L. and J. B. Haddow (1995), "A finite element formulation for finite static axisymmetric deformation of hyperelastic membranes." Computers 85 Structures, 57, 401-405.

Jordan, Peter F (1962), "Stresses and deformations of the thin-walled pressurized torus." Journal of the Aerospace Sciences, 29, 213-225.

Kankanala, S. V. and N. Triantafyllidis (2004), "On finitely strained magnetorheological elastomers." Journal of the Mechanics and Physics of Solids, 52, 2869-2908.

Kanner, Landon M and Cornelius O Horgan (2007), "Elastic instabilities for strain-stiffening rubber-like spherical and cylindrical thin shells under inflation." International Journal of Non-Linear Mechanics, 42, 204-215.

Keh, L. O. S. Co Ting, J. Zang, and X. Zhao (2013), "Magneto-rheological foams capable of tunable energy absorption." In 2013 Proceedings of IEEE Southeastcon, 1-3.

Keplinger, Christoph, Tiefeng Li, Richard Baumgartner, Zhigang Suo, and Siegfried Bauer (2012), "Harnessing snap-through instability in soft dielectrics to achieve giant voltage-triggered deformation." Soft Matter, 8, $285-288$.

Khayat, R. E. and A. Derdouri (1995), "Stretch and inflation of hyperelastic membranes as applied to blow molding." Polymer Engineering and Science, 35, 1852-1863.

Khayat, RE and A Derdouri (1994a), "Inflation of hyperelastic cylindrical membranes as applied to blow moulding. Part I. axisymmetric case." International Journal for Numerical Methods in Engineering, 37, 37733791.

Khayat, RE and A Derdouri (1994b), "Inflation of hyperelastic cylindrical membranes as applied to blow moulding. Part II. non-axisymmetric case." International Journal for Numerical Methods in Engineering, 37, 3793-3808.

Khayat, Roger Edmond, A Derdorri, and Andrés García-Rejón (1992), "Inflation of an elastic cylindrical membrane: non-linear deformation and instability." International journal of solids and structures, 29, 69-87.

Krautz, Maria, David Werner, Mario Schrödner, Alexander Funk, Alexander Jantz, Jana Popp, Jürgen Eckert, and Anja Waske (2017), "Hysteretic behavior of soft magnetic elastomer composites." Journal of Magnetism and Magnetic Materials, 426, 60-63. 
Kydoniefs, A. D. and A. J. M. Spencer (1965), "The finite inflation of an elastic torus." International Journal of Engineering Science, 3, 173-195.

Li, Tiefeng, Christoph Keplinger, Richard Baumgartner, Siegfried Bauer, Wei Yang, and Zhigang Suo (2013), "Giant voltage-induced deformation in dielectric elastomers near the verge of snap-through instability." Journal of the Mechanics and Physics of Solids, 61, 611-628.

Li, Weihua and Xianzhou Zhang (2008), "Research and applications of MR elastomers." Recent Patents on Mechanical Engineering, 1, 161-166.

Li, X. and D. J. Steigmann (1995), "Finite deformation of a pressurized toroidal membrane." International Journal of Non-Linear Mechanics, 30, 583-595.

Maugin, Gérard A and A Cemal Eringen (1972), "Deformable magnetically saturated media. i. field equations." Journal of Mathematical Physics, 13, 143-155.

McMeeking, Robert M and Chad M Landis (2005), "Electrostatic forces and stored energy for deformable dielectric materials." Journal of Applied Mechanics, 72, 581-590.

Naghdi, P. M. and P. Y. Tang (1977), "Large deformation possible in every isotropic elastic membrane." Philosophical Transactions of the Royal Society of London A: Mathematical, Physical and Engineering Sciences, 287, 145-187.

Needleman, Alan (1977), "Inflation of spherical rubber balloons." International Journal of Solids and Structures, 13, 409-421.

Ogden, R. W. and D. J. Steigmann, eds. (2011), Mechanics and Electrodynamics of Magneto- and Electro-Elastic Materials, volume 527. SpringerWeinNew York.

Pao, Y-H (1978), "Electromagnetic forces in deformable continua." In Mechanics today. Volume 4. (A78-35706 14-70) New York, Pergamon Press, Inc., 1978, p. 209-305. NSF-supported research., volume 4, 209-305, New York.

Pao, YH and CS Yeh (1973), "A linear theory for soft ferromagnetic elastic bodies." International Journal of Engineering Science, 11, 415-436.

Papargyri-Beskou, S (2005), "Finite-element analysis of an axisymmetric, thin-walled, nonlinear elastic pressurized torus." Acta Mechanica, 178, 1-22.

Papargyri-Pegiou, S (1995), "Stability of the axisymmetric analytical and numerical solutions in a thin-walled pressurized torus of compressible nonlinear elastic material." International Journal of Engineering Science, 33, 1005-1025.

Papargyri-Pegiou, S and E Stavrakakis (2000), "Axisymmetric numerical solutions of a thin-walled pressurized torus of incompressible nonlinear elastic materials." Computers \& Structures, 77, 747-757.

Patil, Amit, Arne Nordmark, and Anders Eriksson (2014), "Free and constrained inflation of a pre-stretched cylindrical membrane." Proceedings of the Royal Society of London A: Mathematical, Physical and Engineering Sciences, 470, 20140282.

Patil, Amit, Arne Nordmark, and Anders Eriksson (2015), "Instability investigation on fluid-loaded prestretched cylindrical membranes." Proceedings of the Royal Society of London A: Mathematical, Physical and Engineering Sciences, 471, 20150016.

Pearce, S. P., J. R. King, and M. J. Holdsworth (2011), "Axisymmetric indentation of curved elastic membranes by a convex rigid indenter." International Journal of Non-Linear Mechanics, 46, 1128-1138.

Raikher, Yu L, OV Stolbov, and GV Stepanov (2008), "Deformation of a circular ferroelastic membrane in a uniform magnetic field." Technical Physics, 53, 1169-1176.

Sanders, J L and A Liepins (1963), "Toroidal membrane under internal pressure." AIAA Journal, 1, 2105-2110.

Saxena, P., M. Hossain, and P. Steinmann (2013), "A theory of finite deformation magneto-viscoelasticity." International Journal of Solids and Structures, 50, 3886-3897.

Saxena, P., M. Hossain, and P. Steinmann (2014), "Nonlinear magneto-viscoelasticity of transversally isotropic magneto-active polymers." Proceedings of the Royal Society A, 470, 20140082. 
Saxena, Prashant (2017), "Finite deformations and incremental axisymmetric motions of a magnetoelastic tube." Mathematics and Mechanics of Solids, 1081286517697502.

Spain, B. (1965), Tensor Calculus. Oliver and Boyd, London, UK.

Steigmann, D. J. (1990), "Tension-field theory." Proceedings of the Royal Society of London A: Mathematical, Physical and Engineering Sciences, 429, 141-173.

Steigmann, D. J. (2004), "Equilibrium theory for magnetic elastomers and magnetoelastic membranes." International Journal of Non-Linear Mechanics, 39, 1193-1216.

Tamadapu, G. and A. DasGupta (2014a), "Effect of curvature and anisotropy on the finite ination of a hyperelastic toroidal membrane." European Journal of Mechanics-A/Solids, 46, 106-114.

Tamadapu, G., N. N. Dhavale, and A. DasGupta (2013), "Geometrical feature of the scaling behavior of the limit-point pressure of inated hyperelastic membranes." Physical Review E, 88, 053201.

Tamadapu, Ganesh and Anirvan DasGupta (2012), "In-plane surface modes of an elastic toroidal membrane." International Journal of Engineering Science, 60, 25-36.

Tamadapu, Ganesh and Anirvan DasGupta (2013), "Finite inflation analysis of a hyperelastic toroidal membrane of initially circular cross-section." International Journal of Non-Linear Mechanics, 49, 31-39.

Tamadapu, Ganesh and Anirvan DasGupta (2014b), "Finite inflation of a hyperelastic toroidal membrane over a cylindrical rim." International Journal of Solids and Structures, 51, 430-439.

Tews, Alyson M, Kimberly L Pope, and Alan J Snyder (2003), "Pressure-volume characteristics of dielectric elastomer diaphragms." In Smart Structures and Materials, 159-169, International Society for Optics and Photonics.

Tielking, John T (1975), “Analytic tire model. Phase I: The statically loaded toroidal membrane.” Technical report, Transportation Research Institute (UMTRI).

Tiersten, HF (1964), "Coupled magnetomechanical equations for magnetically saturated insulators." Journal of Mathematical Physics, 5, 1298-1318.

Tiersten, HF (1965), "Variational principle for saturated magnetoelastic insulators." Journal of Mathematical Physics, 6, 779-787.

Truesdell, C. and R. Toupin (1960), The Classical Field Theories, 226-858. Springer Berlin Heidelberg, Berlin, Heidelberg.

Wang, Yinling, Yuan Hu, Lin Chen, Xinglong Gong, Wanquan Jiang, Peiqiang Zhang, and Zuyao Chen (2006), "Effects of rubber/magnetic particle interactions on the performance of magnetorheological elastomers." Polymer Testing, 25, 262-267.

Wineman, Alan S (1976), "Large axisymmetric inflation of a nonlinear viscoelastic membrane by lateral pressure." Transactions of The Society of Rheology (195\%-197\%), 20, 203-225.

Xin-chun, Shang and Cheng Chang-jun (1991), "Instability of toroidal membrane with large tensile deformation." Applied Mathematics and Mechanics, 12, 557-564.

Yang, W. H. and W. W. Feng (1970), "On axisymmetrical deformations of nonlinear membranes." Journal of Applied Mechanics, 37, 1002-1011. 